\title{
Decompositions of saturated models of stable theories
}

\author{
by \\ M. C. Laskowski (College Park, MD) and \\ S. Shelah (Jerusalem and New Brunswick, NJ)
}

\begin{abstract}
We characterize the stable theories $T$ for which the saturated models of $T$ admit decompositions. In particular, we show that countable, shallow, stable theories with NDOP have this property.
\end{abstract}

In [7], prior to his work classifying the uncountable models of certain theories, the second author proved a structure theorem for the class of a-models (i.e., $\mathbf{F}_{\kappa_{r}(T)}^{a}$-saturated models in the notation of [7]) of a superstable theory with NDOP. Specifically, in Chapter X of [7] he proved that an a-model of such a theory is a-prime and a-minimal over a normal tree of models, where each node is a-prime over its predecessor and the realization of a regular type. Thus, among superstable theories, the notion of NDOP provides a dichotomy: Either the number of nonisomorphic a-models in each cardinality $\geq 2^{|T|}$ is maximal, or every a-model is determined up to isomorphism by a tree of invariants. It is natural to ask whether a similar dichotomy can be found for the larger class of stable theories. The main obstruction is that an arbitrary stable theory need not have many regular types. Because of this we relax the regularity requirement in Definition 1.7. Our main result, Theorem 1.8, characterizes the stable theories for which large saturated models admit decompositions in this weaker sense.

The first section of the paper states our findings. Section 2 gives some preparatory lemmas that hold for arbitrary stable theories. In Section 3 we work over a single independent tree and characterize when the a-prime model is a-minimal. In Section 4 we prove Theorem 1.8. Finally, in Section 5

2000 Mathematics Subject Classification: 03C45, 03C50.

Key words and phrases: stable theory, saturated model.

Research of M. C. Laskowski partially supported by NSF Grants DMS 0071746 and DMS 0300080.

Research of S. Shelah partially supported by U.S.-Israel Binational Science Foundation Grant no. 2002323 and Israel Science Foundation Grant no. 242/03. Publication no. 851. 
we investigate the effect of restricting to a countable language. By using methods of descriptive set theory we derive unexpected (to us) consequences of NDOP (Theorem 1.11 and Corollary 1.12).

We assume some familiarity with the notions and notational conventions of stability theory, specifically the forking calculus and orthogonality. Knowledge of the material in any of the basic articles or texts in stability (e.g., [1], [5], or [6]) should be sufficient. Also, since many of the arguments that appear here are variants of what occurs in the superstable, NDOP situation, it might be helpful for the reader to skim Chapter X of [7]. We assume that we are working in a large, saturated structure $\mathfrak{C}$ and that our language admits elimination of quantifiers, so the notions of submodel and elementary submodel are interchangeable. To ease notation we do not distinguish between elements of $\mathfrak{C}$ and finite tuples. We write $S(A)$ to denote the union of the Stone spaces $S_{n}(A)$ of complete types over $A$ in $n$ free variables. For brevity we sometimes write $A B$ in place of $A \cup B$.

Acknowledgements. Both authors are extremely grateful to the anonymous referee for spotting several errors and vastly improving the clarity of the exposition.

\section{Statement of results}

HYPOTHESIS. Throughout this paper all theories $T$ are stable and $\kappa$ always denotes the cardinal $\kappa_{r}(T)$.

We work in the category of a-models of $T$. That is, $M$ is an a-model if and only if every type that is almost over a subset of $M$ of size less than $\kappa$ is realized in $M$. An a-model $M$ is a-prime over a set $X$ if $M$ embeds over $X$ into any a-model $N$ that contains $X$. We rely heavily on Theorems IV 3.12 and 4.14 of [7], which assert that a-prime models exist over any set $X$, and are unique up to isomorphism over $X$. An a-model $M$ is a-minimal over $X$ if there is no proper a-submodel of $M$ containing $X$.

We first describe two species of trees of a-models and characterize when the a-prime model over the union of such a tree is in fact a-minimal over the union.

Definition 1.1. A tree $I$ is a nonempty, downward closed subset of $<\omega \delta$ for some ordinal $\delta$. For $\eta, \nu \in I$, we write $\eta \unlhd \nu$ if $\eta$ is an initial segment of $\nu$. For $\eta \neq\langle\rangle, \eta^{-}$denotes the (unique) immediate predecessor of $\eta$.

Definition 1.2. An independent tree of sets is a set $\left\{X_{\eta}: \eta \in I\right\}$ indexed by a tree $I$ such that $X_{\eta} \subseteq X_{\nu}$ whenever $\eta \unlhd \nu$ and $X_{\eta} \underset{X_{\eta^{-}}}{\perp} \bigcup\left\{X_{\nu}: \eta \unlhd \nu\right\}$ for all $\eta \neq\langle\rangle$. We set $X_{J}=\bigcup\left\{X_{\eta}: \eta \in J\right\}$ for any subtree $J \subseteq I$.

An independent tree is normal if, in addition, $\operatorname{tp}\left(X_{\nu} / X_{\eta}\right) \perp X_{\eta^{-}}$for all $\eta, \nu \in I$ satisfying $\eta \neq\langle\rangle$ and $\eta=\nu^{-}$. 
THEOREM 1.3. Let $\left\{M_{\eta}: \eta \in I\right\}$ be any independent tree of a-models and let $M_{I}^{*}$ be a-prime over $M_{I}$. Then the following properties are equivalent:

(i) $M_{I}^{*}$ is a-minimal over $M_{I}$;

(ii) $M_{I}^{*}$ does not contain any infinite indiscernible sequences over $M_{I}$;

(iii) For all nonalgebraic $p \in S\left(M_{I}^{*}\right), p \not \perp M_{\eta}$ for some $\eta \in I$;

(iv) For all types $p$, if $p \not \perp M_{I}^{*}$ then $p \not \perp M_{\eta}$ for some $\eta \in I$.

Two corollaries follow easily from this theorem.

Corollary 1.4. Let $\left\{M_{\eta}: \eta \in I\right\}$ be any independent tree of a-models and let $M_{I}^{*}$ be a-prime over $M_{I}$. If $M_{I}^{*}$ is a-minimal over $M_{I}$ then $M_{J}^{*}$ is a-minimal over $M_{J}$ for any subtree $J \subseteq I$.

Corollary 1.5. Fix a cardinal $\lambda \geq \kappa$. Let $\left\{M_{\eta}: \eta \in I\right\}$ be any independent tree of $\lambda$-saturated models and let $M_{I}^{*}$ be a-prime over $M_{I}$. If $M_{I}^{*}$ is a-minimal over $M_{I}$ then $M_{I}^{*}$ is $\lambda$-saturated.

Next we describe classes of theories $T$ for which a-prime models over certain species of trees are always a-minimal. The strongest such property is the minimality property for independent trees, which asserts that for a given theory $T$, the a-prime model over any independent tree of a-models of $T$ is a-minimal. We say that $T$ has the minimality property for normal trees if this holds for all normal trees. We will see below that these notions coincide.

The following definitions are weakenings of these global notions. They only require that a-prime models be a-minimal for independent trees indexed by some very simple index sets.

Definition 1.6. For $\alpha$ any ordinal, let $I_{\alpha}$ be the tree of height two with a unique root and whose successors are indexed by $\alpha$. In particular, $I_{2}$ denotes the 3-element tree with two incomparable elements. Let $J$ denote the linearly ordered tree of length $\omega$.

A theory $T$ has $N D O P$ if a-prime models over any independent tree of a-models indexed by $I_{2}$ are necessarily a-minimal. For $\mu$ any infinite cardinal, $T$ has $\mu$-NDOP if for all $\alpha<\mu$, every a-prime model over every independent tree of a-models indexed by $I_{\alpha}$ is a-minimal. $T$ has NDIDIP if a-prime models over independent trees of a-models indexed by $J$ are a-minimal. $T$ has normal NDIDIP if a-prime models over a normal tree of a-models indexed by $J$ are a-minimal.

The reader who is disgusted with the phrase "normal NDIDIP" can relax-for stable theories with $\kappa$-NDOP, it is equivalent to NDIDIP.

An easy inductive argument shows that if $T$ has NDOP, then $T$ has $\omega$-NDOP. Additionally, since every type over an a-model is based and stationary over a set of size $<\kappa$, it follows from Theorem 1.3 that if $T$ has $\kappa$-NDOP then $T$ has $\mu$-NDOP for all cardinals $\mu$. In particular, when $T$ is 
superstable the notions of NDOP and $\mu$-NDOP coincide. However, when $T$ is strictly stable there may be a gap between NDOP and $\kappa$-NDOP. It was a surprise to us to discover (see Theorem 1.11) that in fact the gap does not exist when $T$ is countable.

The following notions are central to our attempts at finding invariants for a-models of stable theories.

Definition 1.7. A partial decomposition of an a-model $M$ is a normal tree of a-submodels $\left\{M_{\eta}: \eta \in J\right\}$ of $M$, where $M_{\langle\rangle}$is a-prime over $\emptyset$ and for every $\eta \neq\langle\rangle, M_{\eta}$ is a-prime over $M_{\eta^{-}} \cup\left\{a_{\eta}\right\}$ for some finite tuple $a_{\eta}$. A decomposition of $M$ is a partial decomposition of $M$ such that $M$ is a-prime and a-minimal over $M_{J}$. A partial decomposition is small if $\left|M_{J}^{*}\right|<|M|$. We say that the partial decomposition $\left\{N_{\eta}: \eta \in I\right\}$ extends $\left\{M_{\eta}: \eta \in J\right\}$ simply if $J$ is a subtree of $I$ and $N_{\eta}=M_{\eta}$ for all $\eta \in J$.

THEOREM 1.8. The following are equivalent for a stable theory T:

(i) $T$ has the minimality property for independent trees;

(ii) Every small partial decomposition of every saturated $N$ of size $>2^{|T|}$ extends to a decomposition of $N$;

(iii) $T$ has $\kappa$-NDOP and NDIDIP;

(iv) $T$ has $\kappa$-NDOP and normal NDIDIP;

(v) $T$ has the minimality property for normal trees.

Recall that a tree $I$ is well-founded if it does not have an infinite branch.

Proposition 1.9. Suppose that $T$ has $\kappa-N D O P$ and $\left\{M_{\eta}: \eta \in I\right\}$ is an independent tree of a-models where the index tree $I$ is well-founded. Then every a-prime model over $M_{I}$ is a-minimal over $M_{I}$.

A (stable) theory $T$ is shallow if there is no increasing sequence $\left\langle M_{n}\right.$ : $n \in \omega\rangle$ of a-models of $T$ such that $M_{n+1}$ is a-prime over $M_{n} \cup\left\{a_{n}\right\}$ for some tuple $a_{n}$ for every $n$ and $\operatorname{tp}\left(M_{n+1} / M_{n}\right) \perp M_{n-1}$ for all $n>0$. Clearly, if $T$ is shallow and $\left\{M_{\eta}: \eta \in I\right\}$ is a decomposition of an a-model $M$, then the indexing tree $I$ is well-founded.

COROLlARY 1.10. If T has $\kappa-N D O P$ and is shallow, then $T$ has the minimality property for independent trees. In particular, such a theory satisfies NDIDIP.

Until this point, the cardinality of the language of $T$ was not relevant. By contrast, the countability of $T$ plays a crucial role in the following theorem, as it allows us to employ methods of descriptive set theory (specifically that every analytic subset of a Borel set has the property of Baire).

THEOREM 1.11. If $T$ is countable, then NDOP implies $\omega_{1}$-NDOP (hence $\mu$-NDOP for all cardinals $\mu$ ). 
Our final corollary follows immediately from the two preceding results.

Corollary 1.12. T countable, NDOP, shallow implies NDIDIP.

2. Lemmas about saturation, nonforking and orthogonality. In this section we prove some assorted lemmas about stable theories that will be used in the following sections. The first is an easy characterization of $\lambda$-saturation of models when $\lambda \geq \kappa$.

LEMMA 2.1. Suppose that $\lambda \geq \kappa$ and $M$ is an a-model such that for every subset $A \subseteq M$ with $|A|<\lambda$ and every nonalgebraic $p \in S(A)$, there is a forking extension $q \in S(M)$. Then $M$ is $\lambda$-saturated.

Proof. The definition of an a-model implies that $M$ is $\kappa$-saturated, so assume that $\lambda>\kappa$. Choose any $A \subseteq M$ with $|A|<\lambda$ and choose any nonalgebraic $p \in S(A)$. Clearly, if there is any set $B$ with $A \subseteq B \subseteq M$ and any type $p^{\prime} \in S(B)$ extending $p$ that is algebraic, then $p$ is realized in $M$. But, if we assume by way of contradiction that this is not the case, there would be no difficulty in constructing (by induction on $\alpha$ ) a continuous, increasing sequence $\left\langle A_{\alpha}: \alpha<\kappa\right\rangle$ of subsets of $M$, together with a sequence $\left\langle p_{\alpha}: \alpha<\kappa\right\rangle$ of types, such that $A_{0}=A, p_{0}=p$, each $p_{\alpha} \in S\left(A_{\alpha}\right),\left|A_{\alpha}\right| \leq|A|+\kappa$, and $p_{\beta}$ is a forking extension of $p_{\alpha}$ for all $\alpha<\beta<\kappa$. As stability contradicts the existence of such a sequence, the lemma is proved.

Definition 2.2. Let $\left\{X_{\eta}: \eta \in I\right\}$ be an independent tree of sets. A set $B$ is self-based on $\left\{X_{\eta}: \eta \in I\right\}$ if $\operatorname{tp}\left(B / X_{H}\right)$ does not fork over $B \cap X_{H}$ for all subtrees $H \subseteq I$.

The following lemma is straightforward.

LEMMA 2.3. If $X$ and $A$ are any sets and $|A|<\kappa$, then there is a set $B \supseteq A$ such that $|B|<\kappa, B \backslash A \subseteq X$, and $\operatorname{tp}(B / X)$ does not fork over $B \cap X$. Furthermore, if $B^{\prime} \supseteq B$ and $B^{\prime} \backslash B \subseteq X$, then $\operatorname{tp}\left(B^{\prime} / X\right)$ does not fork over $B^{\prime} \cap X$.

Proof. Given $A$ and $X$, let $C \subseteq X$ be such that $|C|<\kappa$ and $\operatorname{tp}(A / X)$ is based on $C$. Let $B=A \cup C$.

The next lemma is more substantial.

LEMMA 2.4. For every finite index tree $I$, for every independent tree $\left\{X_{\eta}: \eta \in I\right\}$ of models, and for every set $A$ of size $<\kappa$, there is a set $B \supseteq A$ such that $|B|<\kappa, B \backslash A \subseteq X_{I}$, and $B$ is self-based on $\left\{X_{\eta}: \eta \in I\right\}$.

Proof. We argue by induction on $|I|$. If $|I|=1$, this is immediate by Lemma 2.3. So assume that $|I| \geq 2$ and $I=J \cup\left\{\eta^{*}\right\}$, where $\eta^{*}$ is a leaf of $I$. Let $\left\{X_{\eta}: \eta \in I\right\}$ be any independent tree of sets. We assume that the conclusion of the lemma holds for $\left\{X_{\eta}: \eta \in J\right\}$. Fix any set $A$ with $|A|<\kappa$. By Lemma 2.3 choose $B_{0} \supseteq A$ such that $\left|B_{0}\right|<\kappa, B_{0} \backslash A \subseteq X_{I}$, and 
$\operatorname{tp}\left(B_{0} / X_{I}\right)$ does not fork over $B_{0} \cap X_{I}$. Now apply the inductive hypothesis to $B_{0}$ to get $B \supseteq B_{0}$ such that $|B|<\kappa, B \backslash B_{0} \subseteq X_{J}$ (hence $B \backslash A \subseteq X_{I}$ ), and $B$ is self-based on $\left\{X_{\eta}: \eta \in J\right\}$. Finally, by employing Lemma $2.3 \lg \left(\eta^{*}\right)$ times, beginning at $\eta^{*}$ and working downward to \langle\rangle , choose a set $C$ such that $B \cap X_{\eta^{*}} \subseteq C \subseteq X_{\eta^{*}},|C|<\kappa$, and $\operatorname{tp}\left(C / X_{\nu}\right)$ does not fork over $C \cap X_{\nu}$ for all $\nu \unlhd \eta^{*}$.

We argue that the set $B C$ is self-based on $\left\{X_{\eta}: \eta \in I\right\}$. To see this we set some notation. Let $\mu=\left(\eta^{*}\right)^{-}$. For $H \subseteq J$ a subtree, let $H^{\prime}$ be the smallest subtree of $J$ containing $H$ and $\mu$, and let $H^{*}=H \cup\left\{\eta^{*}\right\}$. Note that for any subtree $H \subseteq J, X_{\left(H^{\prime}\right)^{*}}=X_{H^{*}}$ and $X_{H^{*}}=X_{H} X_{\eta^{*}}=X_{H^{\prime}} X_{\eta^{*}}$. Furthermore, since $B \cap X_{\eta^{*}} \subseteq C,\left(B \cap X_{H}\right) \cup C=\left(B \cap X_{H^{\prime}}\right) \cup C$. We begin with the following claim.

Claim. For all subtrees $H \subseteq J, B \underset{\left(B \cap X_{H}\right) C}{\perp} X_{H^{*}}$.

Proof. Fix a subtree $H \subseteq J$. From our observations above we can replace $H$ by $H^{\prime}$ without changing $X_{H^{*}}$ or $\left(B \cap X_{H}\right) C$. Thus, we may assume that $\mu \in H$. Since $X_{I}=X_{J} X_{\eta^{*}}$ and since $\operatorname{tp}\left(B / X_{I}\right)$ does not fork over $B \cap X_{I}$, we have

$$
B \underset{\left(B \cap X_{J}\right)\left(B \cap X_{\left.\eta^{*}\right)}\right.}{\downarrow} X_{J} X_{\eta^{*}}
$$

Let $D=\left(B \cap X_{J}\right) \backslash X_{H}$, so $B \cap X_{J}=D \cup\left(B \cap X_{H}\right)$. Thus

$$
X_{\eta^{*}} \underset{X_{H} D\left(B \cap X_{\eta^{*}}\right)}{\downarrow} B .
$$

Since the tree $\left\{X_{\eta}: \eta \in I\right\}$ is independent, $\operatorname{tp}\left(X_{J} / X_{\eta^{*}}\right)$ does not fork over $X_{\mu}$. Since $\mu \in H$, we have $X_{\mu} \subseteq X_{H}$, so $X_{H} D \underset{X_{\mu}}{\downarrow} X_{\eta^{*}}$, sotp $\left(D / X_{H} X_{\eta^{*}}\right)$ does not fork over $X_{H}$. Combining this with (1) and applying transitivity of nonforking yields

$$
X_{\eta^{*}} \underset{X_{H}\left(B \cap X_{\eta^{*}}\right)}{\downarrow} B .
$$

Since $B$ is self-based on $\left\{X_{\eta}: \eta \in J\right\}, \operatorname{tp}\left(B / X_{H}\right)$ does not fork over $B \cap X_{H}$, so

$$
B \underset{\left(B \cap X_{H}\right)\left(B \cap X_{\eta^{*}}\right)}{\downarrow} X_{H} \text {. }
$$

Transitivity and (2) imply

$$
B_{\left(B \cap X_{H}\right)\left(B \cap X_{\eta^{*}}\right)} X_{H} X_{\eta^{*}},
$$

so the Claim follows since $B \cap X_{\eta^{*}} \subseteq C \subseteq X_{\eta^{*}}$.

Now fix an arbitrary subtree $H \subseteq J$. We will show that $\operatorname{tp}\left(B C / X_{H^{*}}\right)$ does not fork over $(B C) \cap X_{H^{*}}$ and $\operatorname{tp}\left(B C / X_{H}\right)$ does not fork over $(B C) \cap X_{H}$. The former statement follows immediately from the Claim since $(B C) \cap X_{H^{*}}=$ $\left(B \cap X_{H}\right) C$. For the latter, choose the shortest $\nu \unlhd \eta^{*}$ such that $\operatorname{tp}\left(X_{H} / X_{\eta^{*}}\right)$ 
does not fork over $X_{\nu}$. Since $\operatorname{tp}\left(C / X_{\nu}\right)$ does not fork over $C \cap X_{\nu}$ and since $C \subseteq X_{\eta^{*}}, \operatorname{tp}\left(C / X_{H}\right)$ does not fork over $C \cap X_{\nu}$, hence

$$
X_{H} \underset{\left(B \cap X_{H}\right)\left(C \cap X_{\nu}\right)}{\downarrow} C \text {. }
$$

So the Claim and the transitivity of nonforking give

$$
X_{H} \underset{\left(B \cap X_{H}\right)\left(C \cap X_{\nu}\right)}{\downarrow} B C,
$$

which suffices since $(B C) \cap X_{H}=\left(B \cap X_{H}\right) \cup\left(C \cap X_{\nu}\right)$.

Proposition 2.5. Suppose that $\left\{X_{\eta}: \eta \in I\right\}$ is an independent tree of sets with $|I|<\kappa$ and suppose that $|A|<\kappa$. Then there is a set $B \supseteq A$ such that $|B|<\kappa, B \backslash A \subseteq X_{I}$, and $B$ is self-based on $\left\{X_{\eta}: \eta \in I\right\}$.

Proof. When $\kappa=\aleph_{0}$ this is precisely Lemma 2.4, so assume $\kappa>\aleph_{0}$. We begin by inductively constructing an increasing sequence $\left\langle B_{n}: n \in \omega\right\rangle$ of sets, each of size $<\kappa$, such that $B_{0}=A, B_{n} \backslash A \subseteq X_{I}$, and $\operatorname{tp}\left(B_{n} / X_{J}\right)$ does not fork over $B_{n+1} \cap X_{J}$ for all finite subtrees $J \subseteq I$. This is possible by repeated use of Lemma 2.4, since there are fewer than $\kappa$ finite subtrees of $I$.

Let $B^{*}=\bigcup\left\{B_{n}: n \in \omega\right\}$. Since $\kappa$ is regular and uncountable, $\left|B^{*}\right|<\kappa$. We argue that $B^{*}$ is self-based on $\left\{X_{\eta}: \eta \in I\right\}$. Choose an arbitrary subtree $H \subseteq I$ and a finite tuple $b$ from $B^{*}$. To show that $\operatorname{tp}\left(b / X_{H}\right)$ does not fork over $B^{*} \cap X_{H}$, choose a finite tuple $c$ from $X_{H}$ and a formula $\varphi(x, y)$ over $B^{*} \cap X_{H}$ such that $\varphi(b, c)$ holds. In order to show that $\varphi(x, c)$ does not fork over $B^{*} \cap X_{H}$ we show that $\varphi(x, c)$ does not $k$-divide over $B^{*} \cap X_{H}$ for any $k \in \omega$. If, by way of contradiction, $\varphi(x, c)$ did $k$-divide over $B^{*} \cap X_{H}$, then choose $n \in \omega$ and a finite subtree $J \subseteq H$ such that $b \in B_{n}, c \in X_{J}$, and $\varphi(x, y)$ is over $B_{n+1} \cap X_{H}$. If $\left\langle c_{n}: n \in \omega\right\rangle$ were a witness to $\varphi(x, c)$ $k$-dividing over $B^{*} \cap X_{H}$ (i.e., $\operatorname{tp}\left(c_{n} / B^{*} \cap X_{H}\right)=\operatorname{tp}\left(c / B^{*} \cap X_{H}\right)$ for all $n \in \omega$ and $\left\{\varphi\left(x, c_{n}\right): n \in \omega\right\}$ is $k$-inconsistent) then the same sequence would witness $\varphi(x, c) k$-dividing (hence forking) over $B_{n+1} \cap X_{J}$. But this would imply $\operatorname{tp}\left(B_{n} / X_{J}\right)$ forks over $B_{n+1} \cap X_{J}$, which is contrary to our construction of $B_{n+1}$.

Our third group of results uses the ideas in [8] (which in turn were motivated by ideas in [2]) to prove a technical fact (Proposition 2.11) for arbitrary stable theories. Note that there is a much shorter proof of this when $T$ is superstable, which is due to the ubiquity of regular types over a-models.

Definition 2.6. Let $\mathcal{P} \subseteq S(M)$ be a set of types over a model $M$. A set $B$ is weakly dominated by $\mathcal{P}$ over $M$ if there is an independent set $I$ over $M$ consisting of realizations of $\mathcal{P}$ such that $B$ is dominated by $I$ over $M$. (It is possible that $I$ contains many realizations of the same type in $\mathcal{P}$.)

Definition 2.7. Let $M$ be any a-model. A complete type $p$ is an a-type above $M$ if the domain of $p$ is an a-model containing $M$. A class $\mathcal{P}$ of a-types 
above $M$ is $M$-determined if for every $p \in \mathcal{P}$, either $p$ does not fork over $M$ or $p \perp M$. A class $\mathcal{P}$ of a-types above $M$ is dense above $M$ if, for all a-models $N \supseteq M$, every nonalgebraic type over $N$ is nonorthogonal to some element of $\mathcal{P} \cap S(N)$.

Definition 2.8. Let $\mathcal{P}$ be a class of a-types above $M$. A $\mathcal{P}$-sequence over $M$ is a sequence $\left\langle M_{i}, a_{j}: i \leq \alpha, j<\alpha\right\rangle$, where $\left\langle M_{i}: i \leq \alpha\right\rangle$ is an increasing sequence of a-models, $M_{0}=M$, for all $i<\alpha, \operatorname{tp}\left(a_{i} / M_{i}\right) \in \mathcal{P}$ and $M_{i+1}$ is a-prime over $M_{i} \cup\left\{a_{i}\right\}$, and $M_{i}$ is a-prime over $\bigcup_{j<i} M_{j}$ for all limit ordinals $i \leq \alpha$.

Lemma 2.9. If $\mathcal{P}$ is an $M$-determined class of a-types above $M$ and $\left\langle M_{i}, a_{j}: i \leq \alpha, j<\alpha\right\rangle$ is a $\mathcal{P}$-sequence over $M$, then $M_{\alpha}$ is weakly dominated over $M$ by $\left\{\operatorname{tp}\left(a_{j} / M_{j}\right) \mid M: j<\alpha, \operatorname{tp}\left(a_{j} / M_{j}\right)\right.$ does not fork over $\left.M\right\}$.

Proof. Fix an $M$-determined class $\mathcal{P}$ of a-types above $M$. We will prove (by simultaneous induction on $\alpha$ ) that if $\left\langle M_{i}, a_{j}: i \leq \alpha, j<\alpha\right\rangle$ is a $\mathcal{P}$-sequence over $M, I=\left\{a_{j}: \operatorname{tp}\left(a_{j} / M_{j}\right)\right.$ does not fork over $\left.M\right\}$ and $J=\left\{a_{j}: \operatorname{tp}\left(a_{j} / M_{j}\right) \perp M\right\}$, then

(i) $I$ is independent over $M$;

(ii) $M_{\alpha}$ is dominated by $I$ over $M$.

The conclusions are vacuous when $\alpha=0$ and are trivially verified when $\alpha$ is a limit ordinal. So assume that the two conditions hold for the $\mathcal{P}$-sequence $\left\langle M_{i}, a_{j}: i \leq \alpha, j<\alpha\right\rangle$. Choose any $a^{*}$ such that $\operatorname{tp}\left(a^{*} / M_{\alpha}\right) \in \mathcal{P}$ and let $M^{*}$ be a-prime over $M_{\alpha} a^{*}$. We argue that the two conditions also hold for the concatenation of the original $\mathcal{P}$-sequence with $\left\langle M^{*}, a^{*}\right\rangle$. Let $p=\operatorname{tp}\left(a^{*} / M_{\alpha}\right)$.

We first check that (i) continues to hold: If $p \perp M$, then there is nothing to check. On the other hand, if $p$ does not fork over $M$, then $\operatorname{tp}\left(a^{*} / M I\right)$ does not fork over $M$, hence $I \cup\left\{a^{*}\right\}$ is independent over $M$.

We now check that (ii) continues to hold in both cases. First, assume that $p \perp M$. Then if any set $X$ does not fork with $I$ over $M$, then it follows from our inductive assumption that $X$ does not fork with $M_{\alpha}$ over $M$. Since $p \perp M, \operatorname{tp}\left(a^{*} / M_{\alpha} X\right)$ does not fork over $M_{\alpha}$. Since $M^{*}$ is a-prime over $M_{\alpha} a^{*}$, this implies that $X$ does not fork with $M^{*}$ over $M_{\alpha}$. Hence $X$ does not fork with $M^{*}$ over $M$ by transitivity. On the other hand, suppose that $p$ does not fork over $M$. In this case, assume that $X$ does not fork with $I a^{*}$ over $M$. Then, since $I \cup\left\{a^{*}\right\}$ is independent over $M, a^{*} X$ does not fork with $I$ over $M$. By our inductive hypothesis this implies that $a^{*} X$ does not fork with $M_{\alpha}$ over $M$. In particular, $X$ does not fork with $M_{\alpha} a^{*}$ over $M$. So, $X$ does not fork with $M^{*}$ over $M$, since $a^{*}$ dominates $M^{*}$ over $M_{\alpha}$.

Lemma 2.10. Suppose that a class $\mathcal{P}$ of a-types above $M$ is dense above $M$. Then for every $b \in \mathfrak{C}$, there is a $\mathcal{P}$-sequence over $M$ of length $\alpha<\kappa$ such that $b \in M_{\alpha}$. 
Proof. Construct a $\mathcal{P}$-sequence $\left\langle M_{i}, a_{j}: i \leq \alpha, j<\alpha\right\rangle$ over $M$ of maximal length such that $\operatorname{tp}\left(a_{j} / M_{j} b\right)$ forks over $M_{j}$ for every $j<\alpha$. For any such sequence $\operatorname{tp}\left(b / M_{j+1}\right)$ forks over $M_{j}$ for all $j<\alpha$, hence $\alpha<\kappa$. But, since $\mathcal{P}$ is dense above $M$, the only way the process can terminate is if $\operatorname{tp}\left(b / M_{\alpha}\right)$ is algebraic, so $b \in M_{\alpha}$.

Proposition 2.11. Suppose that $\left\{X_{j}: j \in \lambda\right\}$ is any collection of subsets of an a-model $N$. If a type $p$ is not orthogonal to $N$ but $p \perp X_{j}$ for all $j<\lambda$, then there is a type $q \in S(N)$ such that $q \not \perp p$, but $q \perp X_{j}$ for all $j$.

Proof. Choose an a-model $N_{0} \supseteq N$ with $\operatorname{dom}(p) \subseteq N_{0}$ and let $p_{0}$ be the nonforking extension of $p$ to $N_{0}$. Choose $A_{0} \subseteq N_{0}$ of size $<\kappa$ such that $p_{0}$ is definable over $A_{0}$. Choose $C \subseteq N$ of size $<\kappa$ such that $\operatorname{tp}\left(A_{0} / N\right)$ is definable over $C$. Choose a set $\left\{N_{i}: i<\kappa\right\}$ of a-models to be independent over $N$ with $\operatorname{tp}\left(N_{i} / N\right)=\operatorname{tp}\left(N_{0} / N\right)$ for all $i<\kappa$. For each $0<i<\kappa$ choose an automorphism $\sigma_{i}$ of $\mathfrak{C}$ fixing $N$ pointwise and sending $N_{0}$ onto $N_{i}$. Let $A_{i}=\sigma_{i}\left(A_{0}\right)$ and $p_{i}=\sigma_{i}\left(p_{0}\right)$. Since $p_{0} \not \perp N$ it follows that $p_{i} \not \perp p_{j}$ for all $i<j<\kappa$ (see, e.g., 1.4.3.3 of [6]). Let $N^{*}$ be an a-model containing $\bigcup\left\{N_{i}: i<\kappa\right\}$ and let

$\mathcal{P}_{0}=\left\{r: r\right.$ an a-type above $N$ and $\left\{i<\kappa: r \not \perp p_{i}\right\}$ has size $\left.<\kappa\right\}$.

Claim. Some nonalgebraic $q \in S(N)$ is orthogonal to every $r \in \mathcal{P}_{0}$.

Proof. We first argue that $\mathcal{P}_{0}$ is not dense above $N^{*}$. Suppose it were. Let $p_{0}^{+}$denote the nonforking extension of $p_{0}$ to $N^{*}$ and let $b$ be any realization of $p_{0}^{+}$. By Lemma 2.10 there would be a $\mathcal{P}_{0}$-sequence $\left\langle M_{i}, a_{j}: i \leq \alpha, j<\alpha\right\rangle$ over $N^{*}$ of length $\alpha<\kappa$ such that $b \in M_{\alpha}$. For each $j<\alpha$ let $r_{j}=$ $\operatorname{tp}\left(a_{j} / M_{j}\right)$. Since $\alpha<\kappa$ and each $r_{j} \in \mathcal{P}_{0}$ we could find $m<\kappa$ such that $r_{j} \perp p_{m}$ for every $j<\alpha$. But now, if $e$ is any realization of $p_{m}^{+}$(the nonforking extension of $p_{m}$ to $\left.N^{*}\right)$ then we argue by induction on $i \leq \alpha$ that $\operatorname{tp}\left(e / M_{i}\right)$ does not fork over $N^{*}$. In particular, $\operatorname{tp}\left(e / M_{\alpha}\right)$ does not fork over $N^{*}$, hence $p_{m}^{+}$and $p_{0}^{+}$would be almost orthogonal over $N^{*}$. But this would contradict $p_{0} \not \subset p_{m}$ since $N^{*}$ is an a-model.

So $\mathcal{P}_{0}$ is not dense above $N^{*}$. Fix an a-model $N^{\prime} \supseteq N^{*}$ and a nonalgebraic type $q^{\prime} \in S\left(N^{\prime}\right)$ such that $q^{\prime}$ is orthogonal to every $r \in \mathcal{P}_{0} \cap S\left(N^{\prime}\right)$. Choose $D^{\prime}$ of size $<\kappa$ satisfying $C \subseteq D^{\prime} \subseteq N^{\prime}$ over which $q^{\prime}$ is definable and choose $D \subseteq N$ such that there is an automorphism $f$ of $\mathfrak{C}$ fixing $C$ pointwise with $D=f\left(D^{\prime}\right)$. Let $q$ be the nonforking extension of $f\left(q^{\prime} \mid D^{\prime}\right)$ to $S(N)$.

To see that $q$ satisfies the Claim, choose any $r \in \mathcal{P}_{0}$. Say $r \in S\left(N^{\prime \prime}\right)$. Choose any $E \subseteq N^{\prime \prime}$ of size $<\kappa$ on which $r$ is defined, and choose an automorphism $\tau$ of $\mathfrak{C}$ such that $\tau\left|D=f^{-1}\right| D$ (so $\tau$ fixes $C$ pointwise and $\tau(q)$ is parallel to $\left.q^{\prime}\right)$ and $\tau(E) \subseteq N^{\prime}$. Let $r^{\prime} \in S\left(N^{\prime}\right)$ be parallel to $\tau(r)$. Since $E \cup \tau(E)$ is independent of $A_{i}$ over $C$ for almost all $i<\kappa$ (i.e., fewer than $\kappa$ exceptions) and since $r \in \mathcal{P}_{0}$, it follows that $\left\{i<\kappa: \tau(r) \not \perp p_{i}\right\}$ 
has size $<\kappa$, so $r^{\prime} \in S\left(N^{\prime}\right) \cap \mathcal{P}_{0}$. If, by way of contradiction, $q \not \perp r$, then since nonorthogonality is parallelism invariant, it would follow that $q^{\prime} \not \perp r^{\prime}$, contradicting our choice of $q^{\prime}$. Thus $q \perp r$ for all $r \in \mathcal{P}_{0}$.

We argue that any such $q \in S(N)$ satisfies the conclusions of the proposition. Fix such a $q$ and choose any $j<\lambda$. Let $r \in S(N)$ be the nonforking extension of any strong type over $X_{j}$. Since $p \perp X_{j}$ and since $\left\{p_{i}: i \in \kappa\right\}$ are conjugate over $N, r \perp p_{i}$ for all $i$, hence $r \in \mathcal{P}_{0}$. Thus $q \perp r$. That is, $q \perp X_{j}$ for all $j<\lambda$.

It remains to show that $q \not \perp p$. Let $q^{+}$and $p_{i}^{+}(i<\kappa)$ denote the nonforking extensions of $q$ and $p_{i}$ (respectively) to $N^{*}$. Let $\mathcal{P}^{+}=\left\{p_{i}^{+}\right.$: $i<\kappa\}$, let

$$
\begin{aligned}
\mathcal{P} \frac{\perp}{0}=\left\{p \in S\left(N^{*}\right): p \text { is orthogonal to every type } s\right. \text { that is } \\
\text { orthogonal to every type in } \left.\mathcal{P}_{0}\right\}
\end{aligned}
$$

and let

$$
\begin{aligned}
\mathcal{P}_{1}=\left\{s: s \text { is an a-type above } N^{*} \text { such that either } s \perp N^{*}\right. \text { or } \\
\left.\qquad \text { is a nonforking extension of an element of } \mathcal{P}^{+} \cup \mathcal{P}_{0}^{\perp} \perp\right\} .
\end{aligned}
$$

In a moment we will show that $\mathcal{P}_{1}$ is dense above $N^{*}$, but we first show that this suffices. Once it is, then since $\mathcal{P}_{1}$ is $N^{*}$-determined, it follows from Lemmas 2.9 and 2.10 that $q^{+}$is weakly dominated over $N^{*}$ by $\mathcal{P}^{+} \cup \mathcal{P}_{0}^{\perp \perp}$. Since $q^{+}$is nonalgebraic, $q^{+}$(and hence $q$ ) is nonorthogonal to at least one element of $\mathcal{P}^{+} \cup \mathcal{P}_{0}^{\perp \perp}$. Since $q$ is orthogonal to every element of $\mathcal{P}_{0}, q$ is also orthogonal to every element of $\mathcal{P}_{0}^{\perp}$, so $q \not \perp p_{i}$ for some $i<\kappa$. But, since the $p_{i}$ 's are all conjugate over $N$ and since $q \in S(N)$, it follows that $q \not \perp p_{0}$, so $q \not \perp p$.

Thus, it suffices to show that $\mathcal{P}_{1}$ is dense above $N^{*}$. Choose any a-model $M^{\prime} \supseteq N^{*}$ and any nonalgebraic $r \in S\left(M^{\prime}\right)$. We argue that $r$ is nonorthogonal to some element of $\mathcal{P}_{1} \cap S\left(M^{\prime}\right)$. We may assume that $r \not \perp N^{*}$ and $r \perp p_{i}$ for all $i<\kappa$, otherwise $r$ itself would be a witness. We complete the proof by constructing a conjugate type $r^{*} \in \mathcal{P}_{0}^{\perp \perp}$ such that $r \not \perp r^{*}$. To accomplish this, first note that $r \in \mathcal{P}_{0}$, hence $r$ is orthogonal to every type that is orthogonal to every type in $\mathcal{P}_{0}$. Since $r \not \perp N^{*}$, we can choose a type $t \in S\left(N^{*}\right)$ such that $r \not \perp t$. Next, choose sets $D \subseteq M^{\prime}$ and $E \subseteq N^{*}$ such that $|D|<\kappa$, $E=D \cap N^{*}, C \subseteq E, t$ is definable over $E$, and $r$ is definable over $D$. Finally, choose $D^{\prime} \subseteq N^{*}$ such that $D$ and $D^{\prime}$ realize the same strong type over $E$ and are independent over $E$ and let $r^{*} \in S\left(N^{*}\right)$ be definable over $D^{\prime}$ in the same manner that $r$ is over $D$. Since $r \not \perp E, r \not \perp r^{*}$. Also, since $D$ and $D^{\prime}$ realize the same type over $C, r^{*}$ is also orthogonal to every type that is orthogonal to every element of $\mathcal{P}_{0}$. Thus, $r^{*} \in \mathcal{P}_{0}^{\perp \perp}$, so $s$, the nonforking extension of $r^{*}$ to $S\left(M^{\prime}\right)$, is nonorthogonal to $r$ and is in $\mathcal{P}_{1} \cap S\left(M^{\prime}\right)$. 
Our final group of results is aimed at proving Proposition 2.16, which is a variant on the more familiar fact that if $\left\{B_{i}: i \in \kappa\right\}$ are independent over a set $A$ and a stationary type $p$ is nonorthogonal to every $B_{i}$, then $p \not \perp A$. The buildup to the proof of this proposition develops the notion of nonforking in an ultrapower of the monster model. For the rest of this section

Fix a nonprincipal ultrafilter $\mathcal{D}$ on $\omega$ and let $\mathfrak{C}^{*}=\prod \mathfrak{C} / \mathcal{D}$.

We abuse notation slightly and consider $\mathfrak{C}^{*}$ to be an elementary extension of $\mathfrak{C}$. Specifically, we identify an element $a \in \mathfrak{C}$ with the diagonal element $\langle a: i \in \omega\rangle / \mathcal{D} \in \mathfrak{C}^{*}$. For a subset $X \subseteq \mathfrak{C}$ we let $X^{*}$ denote $\prod X / \mathcal{D}$. By our notational convention $X \subseteq X^{*} \subseteq \mathfrak{C}^{*}$.

Lemma 2.12. For any $a \in \mathfrak{C}$ and $B \subseteq \mathfrak{C}, \operatorname{tp}\left(a / B^{*}\right)$ does not fork over $B$.

Proof. Choose any model $M$ such that $B \subseteq M \subseteq \mathfrak{C}$ and $\operatorname{tp}(a / M)$ does not fork over $B$. It clearly suffices to show that $\operatorname{tp}\left(a / M^{*}\right)$ does not fork over $M$. So suppose that $\theta\left(a, b^{*}\right)$ holds (in $\mathfrak{C}^{*}$, where $a$ is identified with its diagonal element) for some formula $\theta(x, y)$ with no hidden parameters. By finite satisfiability, it suffices to find some $b \in M$ such that $\theta(a, b)$ holds. Choose a representation $b^{*}=\left\langle b_{i}: i \in \omega\right\rangle / \mathcal{D}$ with each $b_{i} \in M$. Since $\theta\left(a, b^{*}\right)$ holds, $\left\{i \in \omega: \theta\left(a, b_{i}\right)\right\} \in \mathcal{D}$, so is nonempty.

Lemma 2.13. Suppose that $A \subseteq B_{i} \subseteq \mathfrak{C}$ for all $i \in \omega$, and $\left\{B_{i}: i \in \omega\right\}$ is independent over $A$. Then $\mathfrak{C} \underset{A}{\downarrow} \overline{\bar{B}}$, where $\bar{B}=\prod_{i \in \omega} B_{i} / \mathcal{D}$.

Proof. Choose any $d \in \mathfrak{C}$ and a model $M$ satisfying $A \subseteq M \subseteq \mathfrak{C}$ and $M \underset{A}{\downarrow} \bigcup\left\{B_{i}: i \in \omega\right\} d$. Then $\left\{B_{i}: i \in \omega\right\}$ is independent over $M$ and by transitivity it suffices to prove that $\operatorname{tp}(d / M \bar{B})$ does not fork over $M$. Let $\theta(x, y)$ be an $L(M)$-formula such that $\theta\left(d, b^{*}\right)$ holds for some $b^{*} \in \bar{B}$. By finite satisfiability it suffices to find some $m \in M$ such that $\theta(d, m)$ holds.

Let $E=M \cup\left\{B_{i}: i \in \omega\right\}$. Since $\operatorname{tp}_{\theta}(d / E)$ is definable, there is an $L$-formula $\psi(y, z)$ and an $e \in E$ such that

$$
\theta(d, c) \leftrightarrow \psi(c, e)
$$

for all $c \in E$. Choose a representation $\left\langle b_{i}: i \in \omega\right\rangle / \mathcal{D}$ for $b^{*}$ with $b_{i} \in B_{i}$ for all $i \in \omega$. Since $\theta\left(d, b^{*}\right)$ holds, $\left\{i \in \omega: \theta\left(d, b_{i}\right)\right\} \in \mathcal{D}$. Since $\mathcal{D}$ is nonprincipal and $e$ is finite, and $\left\{B_{i}: i \in \omega\right\}$ is independent over $M$, there is an $i \in \omega$ such that both $\theta\left(d, b_{i}\right)$ holds and $e \underset{M}{\perp} b_{i}$. Since $b_{i} \in E$, (3) implies that $\psi\left(b_{i}, e\right)$ holds. Thus, by symmetry and finite satisfiability there is $m \in M$ such that $\psi(m, e)$ holds. By $(3)$ again, $\theta(d, m)$ holds and we finish.

Lemma 2.14. Suppose that $\left\{a_{i}: i \in \omega\right\} \subseteq \mathfrak{C}, N \subseteq \mathfrak{C}$ is a model, and for each $i \in \omega, M_{i} \subseteq N$ is a model such that $\operatorname{tp}\left(a_{i} / N\right)$ does not fork over $M_{i}$. Then $\operatorname{tp}\left(a^{*} / N^{*}\right)$ does not fork over $\bar{M}$, where $a^{*}=\left\langle a_{i}: i \in \omega\right\rangle / \mathcal{D}$ and $\bar{M}=\prod_{i \in \omega} M_{i} / \mathcal{D}$. 
Proof. First, note that $\bar{M}$ is itself a submodel of $\mathfrak{C}^{*}$. Let $\theta(x, y)$ be any $L$-formula and let $c^{*} \in N^{*}$ be any element such that $\theta\left(a^{*}, c^{*}\right)$ holds. By finite satisfiability it suffices to find $b^{*} \in \bar{M}$ such that $\theta\left(a^{*}, b^{*}\right)$. Choose a representation $\left\langle c_{i}: i \in \omega\right\rangle / \mathcal{D}$ for $c^{*}$ with each $c_{i} \in N$. Let $R=\{i \in \omega$ : $\left.\theta\left(a_{i}, c_{i}\right)\right\}$. Since $\theta\left(a^{*}, c^{*}\right)$ holds, $R \in \mathcal{D}$. We construct a sequence $\left\langle b_{i}: i \in \omega\right\rangle$ as follows: For each $i \in R$, choose $b_{i} \in M_{i}$ such that $\theta\left(a_{i}, b_{i}\right)$. (This is possible since $\operatorname{tp}\left(a_{i} / N\right)$ does not fork over $M_{i}$.) For any $i \notin R$, let $b_{i}$ be an arbitrary element of $M_{i}$. Let $b^{*}=\left\langle b_{i}: i \in \omega\right\rangle / \mathcal{D}$. Then $b^{*} \in \bar{M}$ and $\theta\left(a^{*}, b^{*}\right)$ holds.

We apply these three lemmas in the proof of Proposition 2.16 below.

DeFinition 2.15. Let $\Delta$ be a finite set of (partitioned) $L$-formulas and let $B$ be any set. A stationary type $p$ is $\Delta$-nonorthogonal to $B$, written $p \not \perp^{\Delta} B$, if there is a set $D \supseteq \operatorname{dom}(p) \cup B, \varphi(x, y z) \in \Delta$, and elements $a$ realizing $p \mid D, b \in D$, and $c \in \mathfrak{C}$ such that $\operatorname{tp}(c / D)$ does not fork over $B$, $\varphi(a, b c)$ holds, and $R_{\Delta}(p \mid D \cup\{\varphi(x, b c)\})<R_{\Delta}(p)$.

Clearly, $p \not \perp B$ if and only if $p \not \perp^{\Delta} B$ for some finite $\Delta$. Also, if $B \subseteq B^{\prime}$ and $p \not \perp^{\Delta} B$ then $p \not \perp^{\Delta} B^{\prime}$.

Proposition 2.16. Let $\Delta$ be a finite set of formulas and let $p$ be any stationary type. If $\left\{B_{i}: i \in \omega\right\}$ are independent over $A$ and $p \not \not^{\Delta} B_{i}$ for each $i \in \omega$, then $p \not \perp A$.

Proof. To begin we inductively find submodels $\left\{M_{i}: i \in \omega\right\}$ of $\mathfrak{C}$ such that $B_{i} \subseteq M_{i}$ (hence $p \not \swarrow^{\Delta} M_{i}$ ) for each $i$, yet $\left\{M_{i}: i \in \omega\right\}$ are independent over $A$. For each $i$, choose $D_{i}$ containing $\operatorname{dom}(p) \cup M_{i}$ as in the definition of $\Delta$ nonorthogonality and let $N$ be a substructure of $\mathfrak{C}$ containing $\bigcup\left\{D_{i}: i \in \omega\right\}$. By replacing $p$ by its nonforking extension to $N$, we may assume that $p \in$ $S(N)$. Let $\mathcal{D}$ be any nonprincipal ultrafilter on $\omega$, let $\bar{M}=\prod_{i \in \omega} M_{i} / \mathcal{D}$ and let $N^{*}=\prod N / \mathcal{D}$. It follows immediately from Lemma 2.13 that $N_{A}{ }_{A} \bar{M}$. So, in light of X 1.1 of [7], in order to conclude that $p \not \perp A$ it suffices to show that $p \not \perp \bar{M}$. In fact we will show that $p$ is $\Delta$-nonorthogonal to $\bar{M}$ by demonstrating that $N^{*}$ is a suitable choice of $D$ in Definition 2.15.

Let $a$ be any realization of $p$. It follows from Lemma 2.12 that $a$ realizes the nonforking extension $p^{*}$ of $p$ to $N^{*}$. Let $k=R_{\Delta}(p)=R_{\Delta}\left(p^{*}\right)$. For each $i \in \omega$, since $D_{i} \subseteq N$ we can find $\varphi_{i} \in \Delta, b_{i} \in N$, and $c_{i} \in \mathfrak{C}$ such that $\varphi_{i}\left(a, b_{i} c_{i}\right)$ holds, $\operatorname{tp}\left(c_{i} / N\right)$ does not fork over $M_{i}$ and $R_{\Delta}\left(p \cup\left\{\varphi_{i}\left(x, b_{i} c_{i}\right)\right\}\right)<k$. Since $\Delta$ is finite we may assume that $\varphi_{i}$ is identically $\varphi$ for all $i$. Let $b^{*}=\left\langle b_{i}\right.$ : $i \in \omega\rangle / \mathcal{D}$ and $c^{*}=\left\langle c_{i}: i \in \omega\right\rangle / \mathcal{D}$. Then $b^{*} \in N^{*}$ and $\varphi\left(a, b^{*} c^{*}\right)$ holds. Since $p$ is stationary, its $\Delta$-multiplicity is 1 , hence $\left\{y z: R_{\Delta}(p \cup\{\varphi(x, y z)\})<k\right\}$ is definable. So the Łoś theorem yields

$$
R_{\Delta}\left(p^{*} \cup\left\{\varphi\left(x, b^{*} c^{*}\right)\right\}\right)<k=R_{\Delta}\left(p^{*}\right) .
$$


Finally, since $\operatorname{tp}\left(c_{i} / N\right)$ does not fork over $M_{i}$ for each $i, \operatorname{tp}\left(c^{*} / N^{*}\right)$ does not fork over $\bar{M}$ by Lemma 2.14. So $N^{*}$ witnesses $p \not \chi^{\Delta} \bar{M}$ and we finish.

3. Local minimality: Proofs of 1.3-1.5. In this section we work over a specific independent tree and investigate the consequences of the a-prime model over it being a-minimal. In particular, we prove Theorem 1.3 and two corollaries that follow from it.

Lemma 3.1. Let $\left\{M_{\eta}: \eta \in I\right\}$ be any independent tree of a-models, let $J \subseteq I$ be any subtree, and let $\bar{a}=\left\langle a_{\alpha}: \alpha<\beta\right\rangle$ be any a-construction sequence over $M_{J}$. Then $\bar{a}$ is an a-construction sequence over $M_{I}$ and $\operatorname{tp}\left(\bar{a} / M_{I}\right)$ does not fork over $M_{J}$. In particular, if $M_{J}^{*}$ is a-prime over $M_{J}$, then $M_{J}^{*}$ is the universe of an a-construction sequence over $M_{I}$ and $\operatorname{tp}\left(M_{J}^{*} / M_{I}\right)$ does not fork over $M_{J}$.

Proof. Let $K$ be a maximal subtree such that $J \subseteq K$ and $\operatorname{stp}\left(\bar{a} / M_{J}\right) \vdash$ $\operatorname{stp}\left(\bar{a} / M_{K}\right)$. It follows that $\bar{a}$ is an a-construction sequence over $M_{K}$. By way of contradiction assume that $K \neq I$. Choose $\nu \in K$ and an immediate successor $\eta \in I \backslash K$. Now $M_{K} \frac{\downarrow}{M_{\nu}} M_{\eta}$ and $M_{\nu}$ is an a-model, so, using either $\mathrm{V} 3.2$ of [7] or I 4.3 .4 of [6], an easy induction on $\beta$ shows that $K \cup\{\eta\}$ contradicts the maximality of $K$. The final sentence follows immediately.

Proof of Theorem 1.3. The equivalences (i) $\Leftrightarrow$ (ii) and (iii) $\Leftrightarrow$ (iv) have nothing to do with trees. (i) $\Leftrightarrow$ (ii) is the content of IV 4.21 of [7], (iv) $\Rightarrow$ (iii) is trivial, and (iii) $\Rightarrow$ (iv) follows immediately from Proposition 2.11 (take the sets $X_{i}$ to be the submodels $M_{\eta}$ of $M$ ). The other two implications are generalizations of arguments that appear in the proof of X 2.2 of [7].

(ii) $\Rightarrow$ (iii). Let $r \in S\left(M_{I}^{*}\right)$ be nonalgebraic and assume that $r \perp M_{\eta}$ for all $\eta \in I$. Choose $A \subseteq M_{I}^{*}$ of size less than $\kappa$ over which $r$ is based and stationary. Fix a subtree $J \subseteq I$ of size $<\kappa$ and an a-prime submodel $M_{J}^{*} \subseteq M_{I}^{*}$ that contains $A$. Call a subset $B \subseteq M_{J}^{*}$ suitable if $A \subseteq B,|B|<\kappa$, and $B$ is self-based on $\left\{M_{\eta}: \eta \in J\right\}$. It follows from Proposition 2.5 that for every set $C \subseteq M_{J}^{*}$ of size $<\kappa$, there is a suitable $B$ containing $C$. Thus, by iterating the Claim below $\omega$ times we can construct an infinite Morley sequence $\mathbf{J}$ in $r$ over $A$ inside $M_{I}^{*}$, such that $\operatorname{tp}\left(\mathbf{J} / A M_{I}\right)$ does not fork over $A$. In particular, such a $\mathbf{J}$ is indiscernible over $M_{I}$. So, it suffices to prove the following:

Claim. If $B$ is suitable and $c$ realizes $r \mid B$, then $\operatorname{tp}(c / B) \vdash \operatorname{tp}\left(c / B M_{I}\right)$.

Proof. Fix a suitable $B$ and let $c$ denote any realization of $r \mid B$. We write $B_{\eta}$ for $B \cap M_{\eta}$ and $B_{J^{\prime}}=B \cap M_{J^{\prime}}$ for subtrees $J^{\prime}$ of $J$.

We first argue that $\operatorname{tp}(c / B) \vdash \operatorname{tp}\left(c / B M_{\langle\rangle}\right)$. Choose any finite tuple $a$ from $M_{\langle\rangle}$. Since $B$ is suitable, $\operatorname{tp}(a / B)$ does not fork over $B_{\langle\rangle}$. But $\operatorname{tp}\left(a / B_{\langle\rangle}\right)$ is parallel to a type over $M_{\langle\rangle}$, hence $r$ is orthogonal to $\operatorname{tp}(a / B)$. This implies 
that $a \underset{B}{\downarrow} c$. Since $c$ was an arbitrary realization of $r \mid B$, this implies $\operatorname{tp}(c / B) \vdash$ $\operatorname{tp}(c / B a)$, hence $\operatorname{tp}(c / B) \vdash \operatorname{tp}\left(c / B M_{\langle\rangle}\right)$.

Now let $J^{\prime}$ be a maximal subtree of $J$ such that $\operatorname{tp}(c / B) \vdash \operatorname{tp}\left(c / B M_{J^{\prime}}\right)$. We demonstrate that $J^{\prime}=J$. From the previous paragraph $J^{\prime}$ is nonempty. If $J^{\prime} \neq J$ then there is $\nu \in J \backslash J^{\prime}$ such that its immediate predecessor, denoted by $\eta$, is in $J^{\prime}$. As above, choose $a \in M_{\nu}$. Since we know that $\operatorname{tp}(c / B) \vdash$ $\operatorname{tp}\left(c / B M_{J^{\prime}}\right)$, it suffices to show that $\operatorname{tp}\left(c / B M_{J^{\prime}}\right) \vdash \operatorname{tp}\left(c / B M_{J^{\prime}} a\right)$.

SubClaim. $a \underset{M_{\eta} B_{\nu}}{\downarrow} M_{J^{\prime}} B$.

Proof. Since the original tree is independent, $M_{\nu} \underset{M_{\eta}}{\downarrow} M_{J^{\prime}}$. Since $a B_{\nu} \subseteq$ $M_{\nu}$ this implies

$$
a \underset{M_{\eta} B_{\nu}}{\downarrow} M_{J^{\prime}} B_{\nu} .
$$

However, since $B$ is suitable, $\operatorname{tp}\left(B / M_{J^{\prime}} M_{\nu}\right)$ does not fork over $B_{J^{\prime}} B_{\nu}$. Thus, $B \underset{M_{J^{\prime}} B_{\nu}}{\downarrow} M_{\nu}$. Since $a \in M_{\nu}$, symmetry provides $a \underset{M_{J^{\prime}} B_{\nu}}{\downarrow} B$, so the Subclaim follows from (4) and transitivity.

Now let $p=\operatorname{tp}\left(a / M_{J^{\prime}} B\right)$. The type $p$ does not fork over $M_{\eta} B_{\nu} \subseteq M_{\nu}$, so $p \perp r$. Thus, $\operatorname{tp}\left(c / B M_{J^{\prime}}\right) \vdash \operatorname{tp}\left(c / B M_{J^{\prime}} a\right)$. Hence $J^{\prime}=J$.

We have now established that $\operatorname{tp}(c / B) \vdash \operatorname{tp}\left(c / B M_{J}\right)$. We argue that in fact $\operatorname{tp}(c / B) \vdash \operatorname{tp}\left(c / B M_{I}\right)$. To see this, let $I^{\prime}$ be a maximal subtree that contains $M_{J}$ such that $\operatorname{tp}(c / B) \vdash \operatorname{tp}\left(c / B M_{I^{\prime}}\right)$. As above, if $I^{\prime} \neq I$, then there would be $\nu \in I \backslash I^{\prime}$ whose immediate predecessor $\eta$ is in $I^{\prime}$. Since the tree is independent, $M_{\nu} \underset{M_{\eta}}{\frac{1}{\eta}} M_{I^{\prime}}$. Since $B \subseteq M_{J}^{*}$ and $J \subseteq I^{\prime}$, Lemma 3.1 implies that $B$ is a-constructible, hence a-atomic over $M_{I^{\prime}}$. Since $M_{\eta}$ is a-saturated, $B M_{I^{\prime}}$ is dominated by $M_{I^{\prime}}$ over $M_{\eta}$. Thus, $M_{\nu} \underset{M_{\eta}}{\perp} M_{I^{\prime}} B$. Also, for any finite tuple $a$ from $M_{\nu}, \operatorname{tp}\left(a / M_{\eta}\right) \perp r$. Thus, $a \underset{M_{I^{\prime}} B}{\downarrow} c$ for any such $a$. It follows that $\operatorname{tp}(c / B) \vdash \operatorname{tp}\left(c / B M_{I^{\prime}} M_{\nu}\right)$, contradicting the maximality of $I^{\prime}$. Hence $I^{\prime}=I$ and the proof of (ii) $\Rightarrow$ (iii) is complete.

(iv) $\Rightarrow$ (ii). Let $\mathbf{J} \subseteq M_{I}^{*}$ be a countably infinite, indiscernible sequence over $M_{I}$. By stability, $\mathbf{J}$ is an indiscernible set over $M_{I}$. Partition $\mathbf{J}$ into two infinite sets $\mathbf{J}_{0}$ and $\mathbf{J}_{1}$. Then, by taking $B=\bigcup \mathbf{J}_{0}$ when $\kappa \geq \omega_{1}$ or to be a sufficiently large finite subset of $\mathbf{J}_{0}$ when $\kappa=\omega,|B|<\kappa$ and $\mathbf{J}_{1}$ is an infinite, independent sequence over $B$ such that $\mathbf{J}_{1} \underset{B}{\downarrow} M_{I}$. Let $a \in \mathbf{J}_{1}$ and let $p=\operatorname{tp}(a / B)$. Without loss, we may assume that $p$ is stationary.

Claim. $p \perp M_{\eta}$ for all $\eta \in I$.

Proof. By way of contradiction, choose $\eta$ such that $p \not \perp r$ for some $r \in$ $S\left(M_{\eta}\right)$. Since $p^{(n)}$ is not almost orthogonal to $r^{(n)}$ over $B M_{I}$, we can increase 
$B$ by finitely many elements of $\mathbf{J}_{1}$ and replace $r$ by $r^{(n)}$ and thereby assume that

$$
p \underset{B M_{I}}{\not \not^{a}} r
$$

Choose $A \subseteq M_{\eta}$ of size less than $\kappa$ such that $r$ is based and stationary over $A$. Since $M_{I}^{*}$ is a-prime over $M_{I}$, we can choose $C \subseteq M_{I}$, also of size less than $\kappa$, such that $A \subseteq C$ and $\operatorname{stp}(a B / C) \vdash \operatorname{stp}\left(a B / M_{I}\right)$. Note that this condition implies that

$$
a^{*} B \underset{C}{\downarrow} M_{I}
$$

for any $a^{*}$ such that $\operatorname{tp}\left(a^{*} / B C\right)=\operatorname{tp}(a / B C)$. Since forking is witnessed by a single formula, there is $D$ with $C \subseteq D \subseteq M_{I}$ such that $D \backslash C$ is finite and $\operatorname{tp}(a / B) \frac{\not^{a}}{B D} r$. Since $M_{\eta}$ is a-saturated and $r$ is based and stationary on $A$, there is $e \in M_{\eta}$ such that $\operatorname{tp}(e / A)$ is parallel to $r$ and $\operatorname{tp}(e / B D)$ does not fork over $A$. So, by the non-almost orthogonality condition, there is $a^{*}$ realizing $\operatorname{tp}(a / B)$ such that $a^{*} \underset{B}{\downarrow} D$ and $a^{*} \underset{B D}{\downarrow} e$.

But, since $\operatorname{tp}\left(a / M_{I}\right)$ does not fork and is stationary over $B$, this implies that $a$ and $a^{*}$ have the same type over $B D$, hence over $B C$. So (5) implies that $\operatorname{tp}\left(a^{*} B / M_{I}\right)$ does not fork over $C$. Since $D e \subseteq M_{I}$ this would imply that $\operatorname{tp}\left(a^{*} / B D e\right)$ does not fork over $B D$, which is a contradiction.

Proof of Corollary 1.4. This is straightforward. Fix an independent tree $\left\{M_{\eta}: \eta \in I\right\}$ of a-models such that the a-prime model $M_{I}^{*}$ is a-minimal and fix a subtree $J \subseteq I$. To show that $M_{J}^{*}$ is a-minimal over $M_{J}$ it suffices to show that every nonalgebraic type $p \in S\left(M_{J}^{*}\right)$ is nonorthogonal to some $M_{\eta}$ with $\eta \in J$. So fix such a type $p$. Since $p$ has a nonforking extension to $S\left(M_{I}^{*}\right)$ and since $M_{I}^{*}$ is a-minimal, $p \not \perp M_{\eta}$ for some $\eta \in I$. Choose such an $\eta$ of least length and assume by way of contradiction that $\eta \notin J$. Then $\lg (\eta) \neq 0$ and there is $\nu \unlhd \eta$ of maximal length such that $\nu \in J$. Since the tree is independent, $\operatorname{tp}\left(M_{\eta} / M_{J}\right)$ does not fork over $M_{\nu}$. Since $M_{\nu}$ is an a-model, this implies that $\operatorname{tp}\left(M_{\eta} / M_{J}^{*}\right)$ does not fork over $M_{\nu}$. But then, since $p \perp M_{\nu}$, forking symmetry and X 1.1 of [7] imply that $p \perp M_{\eta}$, which is a contradiction.

Proof of Corollary 1.5. If $\lambda=\kappa$ there is nothing to prove since a-models are $\kappa$-saturated. So fix $\lambda>\kappa$ and an independent tree $\left\{M_{\eta}: \eta \in I\right\}$ of $\lambda$-saturated a-models. Suppose that the a-prime model $M_{I}^{*}$ over $M_{I}$ is aminimal over $M_{I}$. Choose $A \subseteq M$ with $|A|<\lambda$ and choose a nonalgebraic $q \in S(A)$. Because of Lemma 2.1 it suffices to show that $q$ has a forking extension in $S\left(M_{I}^{*}\right)$. Choose a subset $A_{0} \subseteq A$ of size less than $\kappa$ over which $q$ is based and let $q_{0}$ denote the restriction of $q$ to $A_{0}$. By appending a countable Morley sequence in $q_{0}$ to $A_{0}$, we may additionally assume that $q_{0}$ is stationary. Since $M_{I}^{*}$ is a-minimal over $M_{I}, q_{0} \not \perp M_{\eta}$ for some $\eta \in I$. 
Choose $p \in S\left(M_{\eta}\right)$ such that $p \not \perp q_{0}$ and choose $B \subseteq M_{\eta}$ of size less than $\kappa$ over which $p$ is based and stationary. Let $p_{0}$ denote the restriction of $p$ to $B$. Since $p_{0} \not \perp q_{0}$, there is an $n \in \omega$ such that $p_{0}^{(n+1)}$ is not almost orthogonal to $q_{0}^{(n+1)}$ over $B A_{0}$. Since $M_{I}^{*}$ is an a-model, there are finite sequences $C$ and $D$ in $M_{I}^{*}$ realizing $p_{0}^{(n)}$ and $q_{0}^{(n)}$ respectively. Thus,

$$
p_{0} \underset{A_{0} B C D}{\not{ }^{a}} q_{0} \text {. }
$$

Since $M_{\eta}$ is $\lambda$-saturated there is a Morley sequence $\left\langle e_{i}: i \in \lambda\right\rangle$ in $M_{I}^{*}$ of (independent) realizations of $p_{0}$ over $B$ of length $\lambda$. Since $|A B C D|<\lambda$ this implies that $\operatorname{tp}\left(e_{i} / A B C D\right)$ does not fork over $B$ for some $i$. But then $q$ has a forking extension to $S\left(A B C D e_{i}\right)$ and we finish.

4. Global minimality: Proofs of $\mathbf{1 . 8}-\mathbf{1 . 1 0}$. We begin with a definition and a series of lemmas.

Definition 4.1. A partial decomposition $\left\{M_{\eta}: \eta \in J\right\}$ is $\lambda$-full if for every $\eta \in J$ and every nonalgebraic $p \in S\left(M_{\eta}\right)$ satisfying $p \perp M_{\eta^{-}}$(when $\eta \neq\langle\rangle)$ there is a set $H_{\eta} \subseteq J$ of $\lambda$ immediate successors of $\eta$ such that $M_{\nu}$ realizes $p$ for every $\nu \in H_{\eta}$.

The proof of the following lemma is a routine exercise in bookkeeping. (Note that if $\left\{M_{\eta}: \eta \in J\right\}$ is a partial decomposition of $\mathfrak{C}$, then for each $\eta$, $\left|M_{\eta}\right| \leq 2^{|T|}$, so $\left|S\left(M_{\eta}\right)\right| \leq 2^{|T|}$.)

Lemma 4.2. If $\left\{M_{\eta}: \eta \in J\right\}$ is a partial decomposition of $\mathfrak{C}$ and $\lambda \geq$ $2^{|T|}+|J|$, then there is a tree $I$ of size $\lambda$ and a $\lambda$-full partial decomposition $\left\{M_{\eta}: \eta \in I\right\}$ of $\mathfrak{C}$ extending it.

Lemma 4.3. If $|I|=\lambda>2^{|T|},\left\{M_{\eta}: \eta \in I\right\}$ is a $\lambda$-full partial decomposition of $\mathfrak{C}$, and $M_{I}^{*}$ is a-minimal over $M_{I}$, then $M_{I}^{*}$ is $\lambda$-saturated. Moreover, if $\lambda^{<\kappa}=\lambda$, then $M_{I}^{*}$ is saturated of power $\lambda$.

Proof. Fix $A \subseteq M_{I}^{*}$ of size $<\lambda$ and a nonalgebraic, stationary type $p \in S(A)$. We argue that $p$ has a forking extension in $S\left(M_{I}^{*}\right)$.

Let $\mu=|A|+2^{|T|}$. Choose a subtree $J \subseteq I$ with $|J| \leq \mu$ and an a-prime submodel $M_{J}^{*} \preceq M_{I}^{*}$ such that $A \subseteq M_{J}^{*}$. Since $M_{I}^{*}$ is a-minimal, $M_{J}^{*}$ is a-minimal by Corollary 1.4. Thus by Theorem 1.3(iv) we can choose $\eta \in J$ of minimal length such that $p \not \perp M_{\eta}$. By Proposition 2.11, there is $q \in S\left(M_{\eta}\right)$ such that $p \not \perp q$ and $q \perp M_{\eta^{-}}$when $\eta \neq\langle\rangle$.

Let $p^{\prime}, q^{\prime}$ denote the respective nonforking extensions of $p, q$ to $S\left(M_{I}^{*}\right)$. Since $M_{I}^{*}$ is an a-model, $p^{\prime} \frac{\not^{a}}{M_{I}^{*}} q^{\prime}$. Choose a subset $D$ such that $A M_{\eta} \subseteq$ $D \subseteq M_{I}^{*}$ such that $|D| \leq \mu$ and $p^{\prime \prime} \frac{\not}{D}^{a} q^{\prime \prime}$, where $p^{\prime \prime}, q^{\prime \prime}$ denote the respective restrictions of $p, q$ to $D$. Since $\left\{M_{\eta}: \eta \in I\right\}$ is $\lambda$-full and $|D|<\lambda$, there is 
$b \in M_{I}^{*}$ realizing $q^{\prime \prime}$. Thus, $p$ has a forking extension to $M_{I}^{*}$, which implies that $M_{I}^{*}$ is $\lambda$-saturated by Lemma 2.1 .

Finally, since $\left\{M_{\eta}: \eta \in I\right\}$ is $\lambda$-full, $\left|M_{I}\right|=\lambda$. Since $\lambda \geq 2^{|T|}$, the size of an a-prime model over a set of size $\lambda$ has size at most $\lambda^{<\kappa}$. So, if $\lambda^{<\kappa}=\lambda$, then $\left|M_{I}^{*}\right|=\lambda$, hence is saturated.

Lemma 4.4. Fix an independent tree $\left\{M_{\eta}: \eta \in I\right\}$ of a-models. Suppose that $\left\langle J_{\alpha}: \alpha \leq \delta\right\rangle$ is a continuous, increasing sequence of subtrees of I and $\left\langle E_{\alpha}: \alpha<\delta\right\rangle$ is a sequence of sequences such that $E_{\alpha}$ is an a-construction sequence over $M_{J_{\alpha}}$ and $E_{\alpha}$ is an initial segment of $E_{\beta}$ whenever $\alpha<\beta<\delta$. Then any a-prime model over $\bigcup E^{*}$ is a-prime over $M_{J_{\delta}}$, where $E^{*}$ is the shortest sequence such that each $E_{\alpha}$ is an initial segment.

Proof. It follows from Lemma 3.1 that each $E_{\alpha}$ is a-constructible over $M_{J_{\delta}}$, so $E^{*}$ is a-constructible over $M_{J_{\delta}}$ as well. Thus, if $N$ is a-prime (hence a-constructible) over $\bigcup E^{*}$, then $N$ is a-constructible (hence a-prime) over $M_{J_{\delta}}$.

Proof of Theorem 1.8. The implications (i) $\Rightarrow$ (iii) $\Rightarrow$ (iv) as well as (v) $\Rightarrow$ (iv) are trivial.

We begin by showing (iii) $\Rightarrow($ i). Suppose (iii) holds and fix an independent tree of a-models $\left\{M_{\eta}: \eta \in I\right\}$. Let $M_{I}^{*}$ be any a-prime model over $M_{I}$. Form an increasing sequence $\left\langle N_{n}: n \in \omega\right\rangle$ of a-submodels of $M_{I}^{*}$ as follows: For each $n \in \omega$, let $I_{n}=\{\eta \in I: \lg (\eta) \leq n\}$. Let $N_{0}=M_{\langle\rangle}$. We inductively define $N_{n+1}$ as any a-prime submodel of $M_{I}^{*}$ over $N_{n} \cup M_{I_{n+1}}$. Let $N^{*}$ be any a-prime submodel of $M_{I}^{*}$ over $\bigcup\left\{N_{n}: n \in \omega\right\}$. By Lemma 3.1, $N^{*}$ is also a-prime over $M_{I}$, hence $N^{*}$ and $M_{I}^{*}$ are isomorphic over $M_{I}$. So it suffices to show that $N^{*}$ is a-minimal over $M_{I}$. By Theorem 1.3(iii) it suffices to show that every nonalgebraic $p \in S\left(N^{*}\right)$ is nonorthogonal to some $M_{\eta}$. So fix such a nonalgebraic type $p$. By NDIDIP and Theorem 1.3(iii) there is a smallest $n \in \omega$ such that $p \not \subset N_{n}$. If $n=0$ then we finish since $N_{0}=M_{\langle\rangle}$. So assume $n>0$. Let $J_{n}=\{\eta \in I: \lg (\eta)=n\}$. By Lemma 3.1, $\left\{M_{\eta}: \eta \in J_{n}\right\}$ are independent over $N_{n-1}$. Thus, we can find a set $\left\{M_{\eta}^{\prime}: \eta \in J_{n}\right\}$ of submodels of $N_{n}$ such that each $M_{\eta}^{\prime}$ is a-prime over $M_{\eta} \cup N_{n-1}$ and $N_{n}$ is a-prime over $\bigcup\left\{M_{\eta}^{\prime}: \eta \in J_{n}\right\}$. Since $\kappa$-NDOP implies $\mu$-NDOP for any cardinal $\mu$ and since $p \not \perp N_{n}$, it follows from Theorem 1.3(iv) that $p \not \perp M_{\eta}^{\prime}$ for some $\eta \in J_{n}$. But now, since $M_{\eta}$ and $N_{n-1}$ are independent over $M_{\eta^{-}}$, it follows from another instance of NDOP that $p \not \perp M_{\eta}$.

The verification of (iv) $\Rightarrow(\mathrm{v})$ is identical once one checks that if the original tree $M_{I}$ was normal, then the sequence $\left\langle N_{n}: n \in \omega\right\rangle$ defined above is normal as well.

(v) $\Rightarrow$ (ii). Fix a cardinal $\lambda>2^{|T|}$, a saturated model $N$ of size $\lambda$, and a small partial decomposition $\left\{M_{\eta}: \eta \in J\right\}$ of $N$. The existence of a saturated model of size $\lambda \geq 2^{|T|}$ implies that $\lambda^{<\kappa}=\lambda$ (see VIII 4.7 of [7]). Now 
$\left\{M_{\eta}: \eta \in J\right\}$ is also a partial decomposition of $\mathfrak{C}$, so by Lemma 4.2 there is a tree $I$ of size $\lambda$ and a $\lambda$-full partial decomposition $\left\{M_{\eta}: \eta \in I\right\}$ of $\mathfrak{C}$ extending it. By (v), $M_{I}^{*}$ is a-minimal over $M_{I}$, so Lemma 4.3 asserts that $M_{I}^{*}$ is saturated of power $\lambda$. Thus, there is an isomorphism $h: M_{I}^{*} \rightarrow N$ over $M_{J}$. Then $\left\{h\left(M_{\eta}\right): \eta \in I\right\}$ is a decomposition of $N$ extending $\left\{M_{\eta}: \eta \in J\right\}$.

(ii) $\Rightarrow$ (iii). Assume that (ii) holds. The heart of the argument is contained in the proof of the following claim.

Claim. If $\left\{M_{\eta}: \eta \in H\right\}$ is any partial decomposition of $\mathfrak{C}$, then any a-prime model $M_{H}^{*}$ over $M_{H}$ is a-minimal over $M_{H}$.

Proof. Fix $\mu>\left|M_{H}^{*}\right|+2^{|T|}$ such that $\mu^{<\kappa}=\mu$ and choose a saturated model $N$ of size $\mu$ containing $M_{H}^{*}$. By (ii) there is a decomposition $\left\{M_{\eta}\right.$ : $\left.\eta \in H^{\prime}\right\}$ of $N$ extending $\left\{M_{\eta}: \eta \in H\right\}$. Since $N$ is a-minimal over $M_{H^{\prime}}, M_{H}^{*}$ is a-minimal over $M_{H}$ by Corollary 1.4 .

We first verify that NDIDIP holds. Choose an increasing sequence $\left\langle M_{n}\right.$ : $n \in \omega\rangle$ of a-models. Let $M_{\omega}=\bigcup\left\{M_{n}: n \in \omega\right\}$ and let $M_{\omega}^{*}$ be a-prime over $M_{\omega}=\bigcup\left\{M_{n}: n \in \omega\right\}$. We will show that every nonalgebraic type over $M_{\omega}^{*}$ is nonorthogonal to some $M_{n}$. Fix a regular cardinal $\lambda>\left|M_{\omega}^{*}\right|+2^{|T|}$ satisfying $\lambda^{<\kappa}=\lambda$. Note that $\left(\lambda^{+n}\right)^{<\kappa}=\lambda^{+n}$ for each $n \in \omega$. Inductively construct an increasing sequence $\left\langle N_{n}: n \in \omega\right\rangle$ of models such that each $N_{n}$ is saturated of size $\lambda^{+n}$, contains $M_{n}, \operatorname{tp}\left(N_{0} / M_{\omega}^{*}\right)$ does not fork over $M_{0}$, and $\operatorname{tp}\left(N_{n+1} / M_{\omega}^{*} N_{n}\right)$ does not fork over $M_{n+1} N_{n}$ for each $n \in \omega$. It is an easy exercise in nonforking (using X 1.1 of [7]) to see that if a nonalgebraic type in $S\left(M_{\omega}^{*}\right)$ were nonorthogonal to some $N_{n}$, then it would be nonorthogonal to $M_{n}$. So let $N_{\omega}=\bigcup\left\{N_{n}: n \in \omega\right\}$, let $N_{\omega}^{*}$ be a-prime over $N_{\omega}$ and let $p \in S\left(N_{\omega}^{*}\right)$ be nonalgebraic. It suffices to show that $p \not \perp N_{n}$ for some $n \in \omega$.

Let $M_{\langle\rangle} \subseteq N_{0}$ be any a-prime submodel over $\emptyset$. Since $\left\{M_{\langle\rangle}\right\}$is a small, partial decomposition of $N_{0}$, (ii) implies there is an extension $\left\{M_{\eta}: \eta \in J_{0}\right\}$ that is a decomposition of $N_{0}$. Continuing inductively, since a decomposition $\left\{M_{\eta}: \eta \in J_{n}\right\}$ of $N_{n}$ is a small, partial decomposition of the saturated model $N_{n+1}$, (ii) implies that there is an extension $\left\{M_{\eta}: \eta \in J_{n+1}\right\}$ that is a decomposition of $N_{n+1}$.

Let $J_{\omega}=\bigcup\left\{J_{n}: n \in \omega\right\}$. Let $E_{0}$ be an a-construction sequence for $N_{0}$ over $M_{J_{0}}$. By Lemma 3.1, $E_{0}$ is an a-construction sequence over $M_{J_{1}}$, so as $N_{1}$ is both a-prime and a-minimal over $M_{J_{1}}$, there is an a-construction sequence $E_{1}$ end extending $E_{0}$ for $N_{1}$ over $M_{J_{1}}$. Continuing inductively, we construct a sequence $\left\langle E_{n}: n \in \omega\right\rangle$ of sequences such that $E_{n}$ is an a-construction sequence over $M_{J_{n}}$ and $E_{n}$ is an initial segment of $E_{n+1}$ for all $n \in \omega$. By Lemma $4.4, N_{\omega}^{*}$, which was chosen to be a-prime over $N_{\omega}=\bigcup E^{*}$, is also a-prime over $M_{J_{\omega}}$. The Claim above implies that $N_{\omega}^{*}$ is a-minimal over $M_{J_{\omega}}$, so $p \not \perp M_{\eta}$ for some $\eta \in J_{\omega}$. Thus $p \not \perp N_{n}$ for some $n \in \omega$. 
Next we argue that $T$ has $\kappa$-NDOP. Fix any a-model $M$ and any set $\left\{M_{i}: i<\alpha<\kappa\right\}$ of a-models that each contain $M$ and collectively are independent over $M$. Let $M^{*}$ be a-prime over $\bigcup\left\{M_{i}: i<\alpha\right\}$ and choose $\lambda>\left|M^{*}\right|+2^{|T|}$ such that $\lambda^{<\kappa}=\lambda$. Arguing as above, first choose a saturated model $N$ containing $M$ of size $\lambda$ such that $\operatorname{tp}\left(N / M^{*}\right)$ does not fork over $M$ (so $\left\{M_{i}: i<\alpha\right\}$ are independent over $N$ ) and then inductively choose a set $\left\{N_{i}: i<\alpha\right\}$ of saturated models, each of size $\lambda^{+}$such that each $N_{i}$ contains $M_{i} \cup N$ and $\operatorname{tp}\left(N_{i} / M^{*} \cup N \cup\left\{N_{j}: j<i\right\}\right)$ does not fork over $M_{i} \cup N$. Thus $\left\{N_{i}: i<\alpha\right\}$ are independent over $N$. As in the case above, if a type in $S\left(M^{*}\right)$ is nonorthogonal to some $N_{i}$, then it is nonorthogonal to $M_{i}$. So let $N^{*}$ be a-prime over $\bigcup\left\{N_{i}: i<\alpha\right\}$ and fix a nonalgebraic type $p \in S\left(N^{*}\right)$. It is certainly sufficient to show that $p \not \perp N_{i}$ for some $i<\alpha$.

As before, use (ii) to choose a decomposition $\left\{M_{\eta}: \eta \in H\right\}$ of $N$. Then for each $i<\alpha$ use (ii) to get an extension $\left\{M_{\eta}: \eta \in J_{i}\right\}$ that is a decomposition of $N_{i}$. Without loss assume that $J_{i} \cap J_{j}=H$ for all $i \neq j$. Let $I_{i}=H \cup \bigcup\left\{J_{j}\right.$ : $j<i\}$ for each $i<\alpha$ and let $I=\bigcup\left\{I_{i}: i<\alpha\right\}$. Since $\left\{N_{i}: i<\alpha\right\}$ are independent over $N,\left\{M_{\eta}: \eta \in I\right\}$ is a partial decomposition of $\mathfrak{C}$. As in the NDIDIP case above, Lemmas 3.1 and 4.4 imply that $N^{*}$ is a-prime over $M_{I}=$ $\bigcup\left\{M_{\eta}: \eta \in I\right\}$. By the Claim, $N^{*}$ is a-minimal over $M_{I}$. Thus $p \not \perp M_{\eta}$ for some $\eta \in I$ by Theorem 1.3(iii), which implies that $p \not \perp N_{i}$ for some $i<\alpha$.

Proof of Proposition 1.9. Fix a theory $T$ with $\kappa$-NDOP. We recall the usual definition of the depth $\mathrm{dp}_{I}$ of a node $\eta$ of a well-founded tree $I$, namely

$$
\operatorname{dp}_{I}(\eta)=\sup \left\{\operatorname{dp}_{I}(\nu)+1: \nu \text { an immediate successor of } \eta\right\}
$$

and we define the depth of $I$ to be $\operatorname{dp}_{I}(\langle\rangle)$. We prove Proposition 1.9 by induction on the depth of $I$. Fix an ordinal $\alpha$ and assume that every a-prime model over a well-founded, independent tree of a-models of depth less than $\alpha$ is a-minimal over the tree of a-models.

Suppose that $I$ is well-founded of depth $\alpha$ and that $\left\{M_{\eta}: \eta \in I\right\}$ is an independent tree of a-models indexed by $I$. Let $M_{I}^{*}$ be any a-prime model over $M_{I}$ and choose any type $p \not \perp M_{I}^{*}$. We will show that $p \not \perp M_{\eta}$ for some $\eta \in I$, whence $M_{I}^{*}$ is a-minimal over $M_{I}$ by Theorem 1.3. If $I=\{\langle\rangle\}$ then there is nothing to prove. Otherwise, let $A=\{\beta:\langle\beta\rangle \in I\}$. For each $\beta \in A$, let $I(\beta)=\left\{\nu:\langle\beta\rangle^{\wedge} \nu \in I\right\}$ and let $M_{\nu}^{\beta}=M_{\langle\beta\rangle^{\wedge} \nu}$ for each $\nu \in I(\beta)$. Choose $\left\{N_{\beta}: \beta \in A\right\}$ such that each $N_{\beta}$ is an a-prime submodel of $M_{I}^{*}$ over $\bigcup\left\{M_{\nu}^{\beta}: \nu \in I(\beta)\right\}$ and $M_{I}^{*}$ is a-prime over $\bigcup\left\{N_{\beta}: \beta \in A\right\}$. Since the original tree of a-models was independent, $\left\{N_{\beta}: \beta \in A\right\}$ is independent over $M_{\langle\rangle}$. So, since $\kappa$-NDOP implies $\mu$-NDOP for any cardinal $\mu$, we can choose $\beta^{*} \in A$ so that $p \not \perp N_{\beta^{*}}$. By our definition of depth, $\operatorname{dp}\left(I\left(\beta^{*}\right)\right)<\operatorname{dp}(I)=\alpha$, so $N_{\beta}$ is a-minimal over $\bigcup\left\{M_{\nu}^{\beta^{*}}: \nu \in I\left(\beta^{*}\right)\right\}$. So, by Theorem 1.3, $p \not \perp M_{\eta}$ for some $\eta \in I$. 
Proof of Corollary 1.10. Suppose that $T$ has $\kappa$-NDOP and is shallow. Fix any saturated model $N$ with $|N|>2^{|T|}$ and any small partial decomposition $\left\{M_{\eta}: \eta \in J\right\}$ of $N$. We will show that this partial decomposition can be extended to a decomposition of $N$, which suffices by Theorem 1.8. Let $\lambda=$ $|N|$. By VIII 4.7 of [7] the existence of a saturated model of size $\lambda>2^{|T|}$ implies that $\lambda^{<\kappa}=\lambda$. Let $\left\{M_{\eta}: \eta \in I\right\}$ be a $\lambda$-full partial decomposition of $\mathfrak{C}$ extending $\left\{M_{\eta}: \eta \in J\right\}$, which exists by Lemma 4.2. Since $T$ is shallow, the index tree $I$ is well-founded. Since $T$ has $\kappa$-NDOP as well, Proposition 1.9 implies that $M_{I}^{*}$ is a-minimal over $M_{I}$, hence $M_{I}^{*}$ is saturated of power $\lambda$ by Lemma 4.3. So $M_{I}^{*}$ and $N$ are both saturated of size $\lambda$ and contain $M_{J}$. Choose an isomorphism $h: M_{I}^{*} \rightarrow N$ over $M_{J}$. Then $\left\{h\left(M_{\eta}\right): \eta \in I\right\}$ is our desired decomposition of $N$.

5. Countable theories and the proof of Theorem 1.11. Until now, the cardinality of the language was irrelevant. In this section we restrict ourselves to countable languages and prove Theorem 1.11. The assumption of countability allows us to bring in some results from classical descriptive set theory. In particular, the proof given here relies on the fact that analytic subsets of Polish spaces have the property of Baire, i.e., for every analytic $A$ there is an open $U$ such that $A \triangle U$ is meagre (see, e.g., [3]). At its heart, the proof presented here is similar to the argument that every $\boldsymbol{\Sigma}_{1}^{1}$-definable ultrafilter on $\omega$ is principal. The similarity between these two arguments is expounded upon in [4].

THEOREM 5.1. If $T$ is countable and has NDOP, then $T$ has $\mu-N D O P$ for all infinite cardinals $\mu$.

Proof. As noted in the remarks following Definition 1.6, the theorem follows immediately if $T$ is superstable. Consequently, we assume for the whole of this section that

$T$ is countable, stable, but not superstable, with NDOP.

In particular, $\kappa(T)=\aleph_{1}$ and the class of a-models of $T$ is precisely the class of $\aleph_{1}$-saturated models of $T$. The first three subsections provide the requisite background and Theorem 5.1 is proved in Subsection 5.4.

5.1. On stable systems. In this subsection we set notation and prove an extension theorem for stable systems and an embedding theorem for pairs of stable systems.

DEFInition 5.2. A good index set $I$ is a nonempty, countable set of finite sets that is closed under subsets, i.e., $u \in I$ and $v \subseteq u$ implies $v \in I$. An I-system $\bar{X}=\left\{X_{u}: u \in I\right\}$ is a family of sets indexed by $I$ such that 
$X_{u} \subseteq X_{v}$ whenever $u \subseteq v$. For any $I$-system $\bar{X}$ and any $u \in I$, set

$$
X_{\subsetneq u}=\bigcup\left\{X_{v}: v \subsetneq u\right\} \quad \text { and } \quad X_{\nsupseteq u}=\bigcup\left\{X_{v}: v \nsupseteq u\right\}
$$

Throughout this section $\mathbf{J}$ denotes the set of finite subsets of $\omega$ and $K=$ $\{u \in J:|u| \leq 1\}$.

The following notion is the major theme of Section XII.2 of [7].

Definition 5.3. A stable system $\bar{M}$ of models indexed by $I$ is an $I$ system $\bar{M}=\left\{M_{u}: u \in I\right\}$ of models such that $M_{u} \underset{M_{\subsetneq u}}{\downarrow} M_{\nsupseteq u}$ for all $u \in I$.

As a simple special case, note that $\left\{M_{u}: u \in K\right\}$ is a stable system of models if and only if $M_{\emptyset} \subseteq M_{\{i\}}$ for each $i \in \omega$ and $\left\{M_{\{i\}}: i \in \omega\right\}$ are independent over $M_{\emptyset}$.

The following lemma is our primary tool for constructing stable systems.

Lemma 5.4. Suppose that I is a good index set, $u$ finite, $u \notin I$, but every proper subset of $u$ is an element of I. If $\left\{M_{v}: v \in I\right\}$ is a stable system of a-saturated models and $M_{u}$ is a-prime over $M_{\subsetneq u}$, then $\left\{M_{v}: v \in I \cup\{u\}\right\}$ is a stable system of a-saturated models. Moreover, if $M_{u}$ is the union of an a-construction sequence $\bar{a}=\left\langle a_{\alpha}: \alpha<\beta\right\rangle$ over $M_{\subsetneq u}$, then $\bar{a}$ is also an a-construction sequence over $\bigcup\left\{M_{v}: v \in I\right\}$.

Proof. Let $I_{u}=\{v \in I: v \subseteq u\}$. Then $I_{u}$ is a finite, good index set, so by XII, Conclusion 2.11 of [7], $M_{u}$ is $\ell$-isolated over $M_{\subsetneq u}$. Also, by XII, Lemma 2.3(2) of [7], the pair $\left(M_{\subsetneq u}, \bigcup\left\{M_{v}: v \in I\right\}\right)$ satisfies the TarskiVaught property, hence $\operatorname{tp}\left(M_{u} / M_{\subsetneq u}\right)$ has a unique (nonforking) extension to a type in $S\left(\bigcup\left\{M_{v}: v \in I\right\}\right)$ (see, e.g., XII, Lemma 1.12(2) of [7]). In particular, $M_{u} \underset{M_{\subsetneq u}}{\downarrow} M_{\nsupseteq u}$ and the "moreover" clause follows immediately. In order to complete the proof that $\left\{M_{v}: v \in I \cup\{u\}\right\}$ is a stable system it suffices to show that

$$
M_{v} \underset{M_{\subsetneq v}}{\downarrow} M_{\nsupseteq v} M_{u}
$$

where $M_{\not \geq v}=\bigcup\left\{M_{r}: r \in I, v \nsubseteq r\right\}$ for every $v \in I$ satisfying $v \nsubseteq u$ (for $v \subseteq u$ the appropriate requirement is satisfied since $\left\{M_{v}: v \in I\right\}$ is a stable system). So fix $v \nsubseteq u$, hence $M_{\subsetneq u} \subseteq M_{\nsupseteq v}$. From above,

$$
M_{u} \underset{M_{\subsetneq u}}{\downarrow} M_{v} M_{\subsetneq v} M_{\nsupseteq v}
$$

so $M_{v} \underset{M_{\subsetneq u} M_{\subsetneq v} M_{\beth v}}{\downarrow} M_{u}$. Thus $M_{v} \underset{M_{\subsetneq v} M_{\beth v}}{\downarrow} M_{u}$ and the result follows by the transitivity of nonforking.

Proposition 5.5. Suppose $\bar{M}_{K}=\left\{M_{u}: u \in K\right\}$ is a stable system of a-saturated models indexed by $K$ and $M$ is a-prime over $\bigcup \bar{M}_{K}$. Then there is a stable system $\bar{M}_{J}=\left\{M_{u}: u \in J\right\}$ indexed by $J$ such that: 
(i) Each $M_{u} \subseteq M$ and the uth entry of $\bar{M}_{J}=$ the uth entry of $\bar{M}_{K}$ for each $u \in K$

(ii) $M$ is a-prime over $\bigcup \bar{M}_{J}$;

(iii) For each $u \in J \backslash K, M_{u}$ is a-prime over $\bigcup\left\{M_{v}: v \subsetneq u\right\}$;

(iv) For all pairs of good index sets $I \subseteq I^{*} \subseteq J, \bigcup\left\{M_{u}: u \in I^{*}\right\}$ is the union of an a-construction sequence over $\bigcup\left\{M_{u}: u \in I\right\}$.

Proof. Let $\left\langle u_{j}: j \in \omega\right\rangle$ be an enumeration of $J \backslash K$ such that for every $j \in \omega$, if $v \subseteq u_{j}$, then $v \in K \cup\left\{u_{\ell}: \ell<j\right\}$. Define $J_{j}=K \cup\left\{u_{\ell}: \ell<j\right\}$ for each $j \in \omega$. Note that each $J_{j}$ is a good index set. We construct $\mathcal{N}_{J}=\left\{N_{u}\right.$ : $u \in J\}$ as follows. First, let $N_{u}=M_{u}$ for each $u \in K$. Then for each $j \in \omega$ inductively choose $N_{u_{j}}$ to be any a-prime model over $\bigcup\left\{N_{v}: v \subsetneq u\right\}$. Let $N^{*}$ be any a-prime model over $\bigcup \mathcal{N}_{J}$. By successively applying Lemma 5.4 to each of the good index sets $J_{j}$ we find that $\left\{N_{u}: u \in J_{j}\right\}$ is a stable system indexed by $J_{j}$ such that $N_{u_{j}}$ is a-constructible over $\bigcup\left\{N_{v}: v \in J_{j}\right\}$ for every $j \in \omega$. It follows that $\bigcup\left\{N_{u}: u \in J\right\}$ is a-constructible over $\bigcup\left\{N_{u}: u \in K\right\}$. Since $N_{u}=M_{u}$ for all $u \in K$, this implies that $N^{*}$ is a-constructible (hence a-prime) over $\bigcup\left\{M_{u}: u \in K\right\}$. By the uniqueness of a-prime models there is an isomorphism $h: N^{*} \rightarrow M$ fixing $\bigcup\left\{M_{u}: u \in K\right\}$ pointwise. Define $M_{u}=h\left(N_{u}\right)$ for each $u \in J$. It is easy to see that $\bar{M}_{J}=\left\{M_{u}: u \in J\right\}$ satisfies clauses (i)-(iii).

As for (iv), fix good index sets $I \subseteq I^{*} \subseteq J$. Let $\left\langle u_{j}: j<\alpha \leq \omega\right\rangle$ be an enumeration of $I^{*} \backslash I$ such that for every $j<\alpha$, if $v \subseteq u_{j}$, then $v \in I \cup\left\{u_{\ell}: \ell<j\right\}$. Write $I_{j}^{*}=I \cup\left\{u_{\ell}: \ell<j\right\}$ for each $j<\alpha$. Each $I_{j}^{*}$ is a good index set, so it follows from Lemma 5.4 and induction on $j<\alpha$ that $N_{u_{j}}$ is a-constructible over $\bigcup\left\{N_{v}: v \in I_{j}^{*}\right\}$ for each $j$. Clause (iv) follows from this by the transitivity of a-constructibility.

The next definition is not given explicitly in [7], but the notion is inherent in the proof of Lemma XII 2.3 there.

Definition 5.6. Given a good index set $I$ and $* \notin \bigcup I$, let $I^{*}=I \cup$ $\{u \cup\{*\}: u \in I\}$. A linked pair of stable systems $(\bar{A}, \bar{B})$ is a stable system $\bar{C}$ indexed by $I^{*}$ where for each $v \in I^{*}, C_{v}=A_{v}$ when $* \notin v$ and $C_{v}=B_{u}$ when $v=u \cup\{*\}$.

By unraveling the definitions, if $(\bar{A}, \bar{B})$ is a linked pair of stable systems then both $\bar{A}$ and $\bar{B}$ are stable systems indexed by $I, A_{u} \preceq B_{u}$ and $A_{u} \underset{A_{\subsetneq}}{\downarrow} B \nsupseteq u$ for all $u \in I$. Moreover, within the proof of Lemma 2.3 of Chapter XII of [7], the second author shows that these consequences characterize this notion. More precisely, if $\bar{A}, \bar{B}$ are stable systems indexed by $I$ and for each $u \in I, A_{u} \preceq B_{u}$ and $A_{u} \underset{A_{\subsetneq u}}{\downarrow} B_{\nsupseteq u}$, then $(\bar{A}, \bar{B})$ are a linked pair of stable
systems. 
By using this characterization, the proof of the following lemma is just like the proof of the downward Löwenheim-Skolem theorem and is left to the reader.

LEMma 5.7. Let $\bar{M}$ be any stable system of models indexed by $I$ and let $\bar{X}$ be any I-system of sets in which each $X_{u}$ is a countable subset of $M_{u}$. Then there is a stable system $\bar{A}$ such that for each $u \in I, A_{u}$ is countable, $X_{u} \subseteq A_{u} \preceq M_{u}$, and $(\bar{A}, \bar{M})$ is a linked pair of stable systems.

More interesting is Proposition 5.9 below. Its proof uses the following very general lemma, which is also left to the reader.

Lemma 5.8. Suppose $M$ is a-saturated, $A, C$ are countable, $A \subseteq M$, and $q$ is a type in countably many variables over $A C$ that does not fork over $A$. Then $q$ is realized in $M$.

Proposition 5.9. Suppose that $(\bar{A}, \bar{B})$ and $(\bar{A}, \bar{M})$ are both linked pairs of stable systems such that each $B_{u}$ is countable and each $M_{u}$ is a-saturated. Then there is an elementary map $f: \bigcup \bar{B} \rightarrow \bigcup \bar{M}$ such that $f \mid \bigcup \bar{A}=\mathrm{id}$ and $f\left(B_{u}\right) \preceq M_{u}$ for each $u \in I$.

Proof. Fix an enumeration $\left\{u_{j}: j<j^{*} \leq \omega\right\}$ of $I$ such that $u_{i} \subseteq u_{j}$ implies $i \leq j$. To ease notation, write $B_{j}$ in place of $B_{u_{j}}, B_{\subsetneq j}$ in place of $B_{\subsetneq u_{j}}$ and $B_{\nsupseteq j}$ in place of $B_{\nsupseteq u_{j}}$. Note that the condition on our enumeration ensures that $\bigcup\left\{B_{k}: k<j\right\} \subseteq B_{\not j}$. We construct $f$ as the union of a chain of increasing elementary maps

$$
f_{j}: \bigcup \bar{A} \cup \bigcup\left\{B_{k}: k<j\right\} \rightarrow \bigcup \bar{A} \cup \bigcup\left\{M_{k}: k<j\right\}
$$

that satisfy $f_{j} \mid \cup \bar{A}=$ id and $f_{j}\left(B_{k}\right) \preceq M_{k}$ for all $k<j$.

To begin, let $f_{0}$ be the identity map on $\bigcup \bar{A}$. Now assume that $0<j<j^{*}$ and that $f_{j-1}$ has been defined. Since $(\bar{A}, \bar{B})$ is a linked pair of stable systems,

$$
B_{j} \underset{A_{j} B_{\subsetneq j}}{\downarrow} \bigcup \bar{A} B_{\beth_{j}} .
$$

Also, $A_{j} B_{\subsetneq j} \subseteq \operatorname{dom}\left(f_{j-1}\right)$, so $f_{j-1}\left(A_{j} B_{\subsetneq j}\right) \subseteq M_{j}$. Since $M_{j}$ is a-saturated, Lemma 5.8 ensures the existence of an elementary map $f_{j} \supseteq f_{j-1}$ with $f_{j}\left(B_{j}\right) \preceq M_{j}$, and our proof is complete.

5.2. Pseudo $\ell$-isolation. If the index set $I$ is finite and $\bar{M}=\left\{M_{u}: u \in I\right\}$ is a stable system of a-saturated models, then a type $p \in S\left(\bigcup\left\{M_{u}: u \in I\right\}\right)$ is a-isolated if and only if it is $\ell$-isolated (see XII 2.11 of [7]). When one is analyzing a type over the union of a stable system of models of a superstable theory, the restriction that $I$ be finite is inconsequential since the type is based on the union of a finite subsystem. However, here our theory is strictly stable, so we need an analogue of this result that holds for stable systems over infinite index sets as well. The notion of pseudo $\ell$-isolation satisfies our needs. 
Definition 5.10. A formula $\psi(x)$ (possibly with hidden parameters) decides the formula $\varphi(x, e)$ if either $\psi(x) \vdash \varphi(x, e)$ or $\psi(x) \vdash \neg \varphi(x, e)$. For any model $M, \psi(x)$ decides $\varphi(x, M)$ if $\psi(x)$ decides $\varphi(x, e)$ for all $e \in M$.

Lemma 5.11. Suppose $M \subseteq A, M$ is an a-saturated model, and $p \in S(A)$ is an a-isolated type. Then for any $L$-formula $\varphi(x, y)$ there is $\psi(x) \in p$ that decides $\varphi(x, M)$.

Proof. Fix an $L$-formula $\varphi(x, y)$. Since $p$ is a-isolated, we can choose $q=\left\{\psi_{n}(x): n<n^{*} \leq \omega\right\} \subseteq p$ such that $q \vdash p$ and $\psi_{n} \vdash \psi_{n-1}$ for all $0<n<n^{*}$. For each $n<n^{*}$ let

$$
Z_{n}=\left\{e \in M: \psi_{n} \text { decides } \varphi(x, e)\right\} .
$$

Since $T$ is stable, each $Z_{n}$ is $M$-definable. Furthermore, since $q \vdash p$ and $p \in S(A)$ is a complete type, $\bigcup_{n \in \omega} Z_{n}=M$. Since $M$ is a-saturated, this implies $M=Z_{m}$ for some $m<n^{*}$. That is, $\psi_{m}$ decides $\varphi(x, M)$.

Definition 5.12. Suppose that $\bar{M}$ is an $I$-system of models. A type $p \in S(\bigcup \bar{M})$ is pseudo -isolated over $\bar{M}$ (not over $\bigcup \bar{M}$ !) if for every $u \in I$ and every $L$-formula $\varphi(x, y)$, there is $\psi(x) \in p$ deciding $\varphi\left(x, M_{u}\right)$.

A set $D$ is pseudo $\ell$-atomic over $\bar{M}$ if $\operatorname{tp}(d / \bigcup \bar{M})$ is pseudo $\ell$-isolated over $\bar{M}$ for all finite tuples $d$ from $D$.

The following lemma connects these notions with a-atomicity.

Lemma 5.13. Let $\bar{M}$ be an I-system of a-saturated models. For any set $D, D$ is a-atomic over $\bigcup \bar{M}$ if and only if $D$ is pseudo $\ell$-atomic over $\bar{M}$.

Proof. Left to right is immediate by Lemma 5.11. For the converse let $p \in S(\bigcup \bar{M})$ be pseudo $\ell$-isolated over $\bar{M}$. For each $L$-formula $\varphi(x, y)$ and each $u \in I$, choose $\psi_{\varphi, u}(x) \in p$ that decides $\varphi\left(x, M_{u}\right)$. Then $q=\left\{\psi_{\varphi, u}(x)\right.$ : $\varphi, u\}$ witnesses that $p$ is a-atomic over $\bigcup \bar{M}$.

Lemma 5.14. Suppose that $I$ is a good index set that is closed under unions, i.e., $u, v \in I$ implies $u \cup v \in I$. Let $\bar{M}$ and $\bar{M}^{\prime}$ be I-systems such that $M_{u} \subseteq M_{u}^{\prime}$ and $\operatorname{tp}\left(M_{u}^{\prime} / \bigcup \bar{M}\right)$ is finitely satisfiable in $M_{u}$ for all $u \in I$. If $p \in S(\bigcup \bar{M})$ is pseudo $\ell$-isolated over $\bar{M}$ then $p$ has a unique extension to $p^{\prime} \in S\left(\bigcup \bar{M}^{\prime}\right)$ (which is pseudo l-isolated over $\left.\bar{M}^{\prime}\right)$.

Proof. For each $\varphi(x, y)$ and $u \in I$ choose $\psi(x) \in p$ that decides $\varphi\left(x, M_{u}\right)$. We argue that $\psi(x)$ decides $\varphi\left(x, M_{u}^{\prime}\right)$ as well. To see this, choose $v \in I$ such that $\psi(x)$ is over $M_{v}$. By our constraint on $I$ we may assume that $u \subseteq v$. If $\psi\left(x, a_{v}\right)$ did not decide $\varphi\left(x, M_{u}^{\prime}\right)$ then for some $b \in M_{u}^{\prime}, \theta\left(a_{v}, b\right)$ would hold, where $\theta(y, z)$ is $\exists x_{1} \exists x_{2}\left[\psi\left(x_{1}, y\right) \wedge \psi\left(x_{2}, y\right) \wedge\left(\varphi\left(x_{1}, z\right) \nLeftarrow \varphi\left(x_{2}, z\right)\right)\right]$. But then finite satisfiability would imply that $\theta\left(a_{v}, a_{u}\right)$ would hold for some $a_{u} \in M_{u}$, which would contradict the fact that $\psi(x)$ decides $\varphi\left(x, M_{u}\right)$. Thus 
$\left\{\psi_{\varphi, u}(x): \varphi, u\right\}$ has a unique extension to $p^{\prime} \in S\left(\bigcup \bar{M}^{\prime}\right)$ and the same formulas witness the pseudo $\ell$-isolation of $p^{\prime}$.

If $\bar{M}=\left\{M_{u}: u \in J\right\}$ is a stable system of models indexed by $J$ and $X \subseteq \omega$, let $\bar{M}_{X}$ be the stable system (also indexed by $\left.J\right)\left\{M_{u \cap X}: u \in J\right\}$, while $M_{X}$ denotes the model with universe $\bigcup\left\{M_{u}: u \in J \cap \mathcal{P}(X)\right\}$. The fact that $M_{X}$ is a model follows from the fact that the index set $J$ is closed under finite unions. It is readily checked that $M_{X}=\bigcup \bar{M}_{X}$. In particular, $M_{\omega}=\bigcup \bar{M}$. The following lemma is a stable system analogue of Lemma 3.1.

Lemma 5.15. Let $\bar{M}$ be any stable system of a-saturated models indexed by $J$, let $X \subseteq \omega$ and let $Y=\omega \backslash X$.

(i) If $D$ is pseudo $\ell$-atomic over $\bar{M}_{X}$ then $\operatorname{tp}\left(D / M_{X}\right)$ has a unique extension to a type over $M_{\omega}$, and $D$ is also pseudo $\ell$-atomic over $\bar{M}$.

(ii) Every a-construction sequence $\bar{a}=\left\langle a_{\alpha}: \alpha<\beta\right\rangle$ over $M_{X}$ is an aconstruction sequence over $M_{\omega}$ and $\operatorname{tp}\left(\bar{a} / M_{\omega}\right)$ does not fork over $M_{X}$.

(iii) If $N_{X}, N_{Y}$ are a-prime over $M_{X}, M_{Y}$ respectively, then

(a) $\operatorname{tp}\left(N_{X} / M_{X}\right) \vdash \operatorname{tp}\left(N_{X} / M_{\omega} N_{Y}\right)$,

(b) $\operatorname{tp}\left(M_{\omega} / M_{X} M_{Y}\right) \vdash \operatorname{tp}\left(M_{\omega} / N_{X} N_{Y}\right)$.

Proof. (i) Since $\bar{M}$ is a stable system, $\operatorname{tp}\left(M_{u} / M_{X}\right)$ is finitely satisfiable over $M_{u \cap X}$, so we can apply Lemma 5.14 to the stable systems $\bar{M}_{X}$ and $\bar{M}$.

Using (i) and Lemma 5.13, (ii) follows by induction on $\beta$.

For both parts of (iii) choose $\varphi(x, y, z)$ and tuples $c_{1}$ from $N_{X}, c_{2}$ from $N_{Y}$, and $d$ from $M_{\omega}$ such that $\varphi\left(c_{1}, c_{2}, d\right)$ holds.

We first show that there is $\psi(x, e) \in \operatorname{tp}\left(c_{1} / M_{X}\right)$ such that $\psi(x, e) \vdash$ $\varphi\left(x, c_{2}, d\right)$. Choose a finite $u$ such that $d$ is from $M_{u}$. Since $\bar{M}_{X}$ is a stable system and $N_{X} / M_{X}$ is a-atomic, $\operatorname{tp}\left(c_{1} / M_{X}\right)$ is pseudo $\ell$-isolated over $\bar{M}_{X}$. So there is $\psi(x, e) \in \operatorname{tp}\left(c_{1} / M_{X}\right)$ such that $\psi(x, e) \vdash \operatorname{tp}_{\varphi}\left(c_{1} / M_{u \cap X}\right)$. We argue that this $\psi(x, e) \vdash \varphi\left(x, c_{2}, d\right)$.

Let $Z=Y \cup u$. Since $X \cap Z=u \cap X$ and since $\bar{M}$ is a stable system, $\operatorname{tp}\left(M_{Z} / M_{X}\right)$ does not fork over $M_{u \cap X}$. As well, $\operatorname{tp}\left(c_{2} / M_{Y}\right)$ is a-isolated, hence $\operatorname{tp}\left(c_{2} / M_{Z}\right)$ is a-isolated as in (ii). Since $M_{u \cap X}$ is an a-model, $c_{2} M_{Z}$ is dominated by $M_{Z}$ over $M_{u \cap X}$, hence $M_{X} \underset{M_{u \cap X}}{\downarrow} M_{Z} c_{2}$ follows by symmetry. Since $M_{u \cap X}$ is a model, the pair $\left(M_{u \cap X}, M_{Z} c_{2}\right)$ has the Tarski-Vaught property, hence $\operatorname{tp}_{\varphi}\left(c_{1} / M_{u \cap X}\right)$ has a unique extension $q_{\varphi} \in S_{\varphi}\left(M_{Z} c_{2}\right)$ and $\psi(x, e) \vdash q_{\varphi}$. In particular, $\psi(x, e)$ decides $\varphi\left(x, c_{2}, d\right)$. But since $\varphi\left(c_{1}, c_{2}, d\right)$ holds, it decides it positively, i.e., $\psi(x, e) \vdash \varphi\left(x, c_{2}, d\right)$. Thus, (iii)(a) holds.

To establish (iii)(b) choose $d^{\prime}$ such that $\operatorname{tp}\left(d^{\prime} / M_{X} M_{Y}\right)=\operatorname{tp}\left(d / M_{X} M_{Y}\right)$. It suffices to show that $\varphi\left(c_{1}, c_{2}, d\right)$ holds. So choose $\psi(x, e)$ as above and let

$$
\theta(y, d, e):=\forall x[\psi(x, e) \rightarrow \varphi(x, y, d)]
$$


By our choice of $\psi(x, e), \theta(y, \bar{d}, e) \in \operatorname{tp}\left(c_{2} / M_{\omega}\right)$. By (i), $\operatorname{tp}\left(c_{2} / M_{Y}\right) \vdash$ $\operatorname{tp}\left(c_{2} / M_{\omega}\right)$, so there is $\delta\left(y, e^{\prime}\right) \in \operatorname{tp}\left(c_{2} / M_{Y}\right)$ such that $\delta\left(y, e^{\prime}\right) \vdash \theta(y, d, e)$. Since $e, e^{\prime} \in M_{X} \cup M_{Y}$, it follows that $\delta\left(y, e^{\prime}\right) \vdash \theta\left(y, d^{\prime}, e\right)$, hence $\theta\left(c_{2}, d^{\prime}, e\right)$. Thus, $\varphi\left(c_{1}, c_{2}, d^{\prime}\right)$ holds as required.

5.3. The standard topology on $\mathcal{P}(\omega)$. The standard topology on $\mathcal{P}(\omega)$ is obtained by positing that the sets

$$
U_{F, G}=\{X \in \mathcal{P}(\omega): F, G \text { are finite subsets of } \omega, F \subseteq X, X \cap G=\emptyset\}
$$

form a basis of open sets. Topologized in this way, the natural mapping between subsets of $\omega$ and characteristic functions is a homeomorphism between $\mathcal{P}(\omega)$ and the Cantor set ${ }^{\omega} 2$.

Note that $U_{F, G}=\emptyset$ if and only if $F \cap G \neq \emptyset$. Let $\mathcal{D}=\{(F, G): F, G$ are finite subsets of $\omega$ and $F \cap G=\emptyset\}$. For $(F, G),\left(F^{\prime}, G^{\prime}\right) \in \mathcal{D}$ we write $(F, G) \leq\left(F^{\prime}, G^{\prime}\right)$ if and only if $F \subseteq F^{\prime}$ and $G \subseteq G^{\prime}$.

It is easily checked that a set $R \subseteq \mathcal{P}(\omega)$ is nowhere dense if and only if for every $(F, G) \in \mathcal{D}$ there is $\left(F^{\prime}, G^{\prime}\right) \in \mathcal{D}$ such that $\left(F^{\prime}, G^{\prime}\right) \geq(F, G)$ and $U_{F^{\prime}, G^{\prime}} \cap R=\emptyset$. Recall that a set $Z \subseteq \mathcal{P}(\omega)$ is meagre if it is a countable union of nowhere dense subsets.

The following lemma is routine, but is included for completeness.

Lemma 5.16. Let $Z$ be any meagre subset of $\mathcal{P}(\omega)$. Then:

(i) There is $X \in \mathcal{P}(\omega)$ such that $X, \omega \backslash X \notin Z$.

(ii) There are $\left\{X_{i}: i \in \omega\right\} \subseteq \mathcal{P}(\omega) \backslash Z$ with $X_{i} \cap X_{j}=\emptyset$ when $i<j<\omega$.

Proof. Suppose that $Z=\bigcup_{n \in \omega} R_{n}$, where each $R_{n}$ is nowhere dense.

(i) Using the characterization of nowhere denseness given above, inductively construct a sequence $\left\langle\left(F_{n}, G_{n}\right): n \in \omega\right\rangle$ from $\mathcal{D}$ that satisfies $\left(F_{n}, G_{n}\right) \leq\left(F_{n+1}, G_{n+1}\right), U_{F_{2 n}, G_{2 n}} \cap R_{n}=\emptyset$, and $U_{G_{2 n+1}, F_{2 n+1}} \cap R_{n}=\emptyset$. Take $X=\bigcup_{n \in \omega} F_{n}$. Then $X \in U_{F_{n}, G_{n}}$ for all $n$, so $X \notin Z$. Furthermore, $\omega \backslash X \in U_{G_{n}, F_{n}}$ for all $n$, so $\omega \backslash X \notin Z$ as well.

(ii) Fix a bijection $\Phi: \omega \rightarrow \omega \times \omega$. Call an $\omega$-sequence $\mathcal{F}=\left\langle F_{i}: i \in \omega\right\rangle$ of (finite) subsets of $\omega$ an approximating sequence if $\left\{F_{i}: i \in \omega\right\}$ are pairwise disjoint and $\bigcup\left\{F_{i}: i \in \omega\right\}$ is finite. We say that an approximating sequence $\mathcal{F}=\left\langle F_{i}: i \in \omega\right\rangle$ satisfies Condition $k$ if, writing $\Phi(k)=(i, j)$, we have

$$
U_{F_{i}, G_{i}} \cap R_{j}=\emptyset \quad \text { where } \quad G_{i}=\bigcup\left\{F_{l}: l \neq i\right\} .
$$

We inductively construct approximating sequences $\mathcal{F}^{n}=\left\langle F_{i}^{n}: i \in \omega\right\rangle$ for each $n \in \omega$ such that $F_{i}^{n} \subseteq F_{i}^{m}$ for all $i$ and all $n<m<\omega$ and $\mathcal{F}^{n}$ satisfies Condition $k$ for all $k<n$.

To start, define $\mathcal{F}^{0}=\left\langle F_{i}^{0}: i \in \omega\right\rangle$, where each $F_{i}^{0}=\emptyset$. Now assume that $\mathcal{F}^{n}$ has been defined and let $\Phi(n)=\left(i^{*}, j^{*}\right)$. Let $G=\bigcup\left\{F_{l}^{n}: l \neq i^{*}\right\}$. Since $R_{j^{*}}$ is nowhere dense, there is $\left(F^{\prime}, G^{\prime}\right) \geq\left(F_{i^{*}}^{n}, G\right)$ such that $U_{F^{\prime}, G^{\prime}} \cap R_{j^{*}}=\emptyset$. Let $m$ be any integer $\neq i^{*}$ such that $F_{m}^{n}=\emptyset$. Let $F_{i^{*}}^{n+1}=F^{\prime}, F_{m}^{n+1}=G^{\prime} \backslash G$, 
and $F_{l}^{n+1}=F_{l}^{n}$ for all $l \neq i^{*}, m$. Then $\mathcal{F}^{n+1}=\left\langle F_{i}^{n+1}: i \in \omega\right\rangle$ satisfies Condition $k$ for all $k \leq n$.

Finally, for each $i \in \omega$ take $X_{i}=\bigcup\left\{F_{i}^{n}: n \in \omega\right\}$.

5.4. Proof of Theorem 5.1. By the remarks following Definition 1.6, it suffices to show that $T$ has $\omega_{1}$-NDOP. Choose a family $\left\{M_{i}: i<\omega\right\}$ of a-saturated models that contain and are independent over a common asaturated model $M_{\emptyset}$, and let $M$ be a-prime over $\bigcup\left\{M_{i}: i<\omega\right\}$. Fix a nonalgebraic type $p \in S(M)$. We will eventually show that $p$ is nonorthogonal to some $M_{i}$, which suffices by Theorem 1.3.

Let $\bar{M}_{K}$ denote the stable system indexed by $K$, where $M_{\{i\}}=M_{i}$ for each $i \in \omega$. Choose a stable system $\bar{M}_{J}=\left\{M_{u}: u \in J\right\}$ extending $\bar{M}_{K}$ satisfying clauses (i)-(iv) of Proposition 5.5.

We adopt the notation prior to Lemma 5.15 for the whole of this section, not only for the stable system $\bar{M}$ in the claim below, but also for the related systems $\bar{A}$ and $\bar{B}$ that follow. For each $X \subseteq \omega$ let

$\mathcal{N}_{X}=\left\{N \preceq M: N\right.$ is a-prime over $M_{X}$ and $M$ is a-prime over $\left.N \cup M_{\omega}\right\}$.

For each finite $\Delta \subseteq L$, let $W_{\Delta}=\left\{X \subseteq \omega: p \not \underline{\perp}^{\Delta} N\right.$ for some $\left.N \in \mathcal{N}_{X}\right\}$ and let $W=\bigcup\left\{W_{\Delta}: \Delta \subseteq L\right.$ finite $\}$.

Claim 5.17. For all $X \subseteq \omega$, at least one of $X, \omega-X$ is in $W$.

Proof. Fix $X \subseteq \omega$ and let $Y=\omega \backslash X$. Let $N_{X}, N_{Y}$ be a-prime models over $M_{X}, M_{Y}$ respectively. Let $M^{*}$ be a-prime over $N_{X} \cup \mathcal{N}_{Y} \cup M_{\omega}$. We argue that $M^{*}$ is also a-prime over each of the four sets $M_{\omega}, N_{X} \cup M_{\omega}, N_{Y} \cup M_{\omega}$, and $N_{X} \cup N_{Y}$.

To see this, first note that by applying Proposition 5.5(iv) with $I=$ $(\mathcal{P}(X) \cup \mathcal{P}(Y)) \cap J$ and $I^{*}=J, M_{\omega}$ is a-constructible over $M_{X} \cup M_{Y}$. By Lemma 5.15(iii)(b), $M_{\omega}$ is a-constructible over $N_{X} \cup N_{Y}$. Thus, $M^{*}$ is a-constructible (hence a-prime) over $N_{X} \cup N_{Y}$. As well, by Lemma 5.15(ii), $N_{X}$ is a-constructible over $M_{\omega}$. By Lemma 5.15(iii)(a), $N_{Y}$ is a-constructible over $M_{\omega} \cup N_{X}$. Hence $M^{*}$ is a-prime over $M_{\omega}$ as well as over $M_{\omega} \cup N_{X}$. That $M^{*}$ is a-prime over $M_{\omega} \cup N_{Y}$ is symmetric.

But now, recall that $M$ is also a-prime over $M_{\omega}$. So there is an isomorphism $h: M^{*} \rightarrow M$ fixing $M_{\omega}$ pointwise. Since $\bar{M}$ is a stable system, $M_{X} \underset{M_{\emptyset}}{\frac{1}{\square}} M_{Y}$. Also, $h\left(N_{X}\right)$ is a-prime over $M_{X}$, hence dominated by $M_{X}$ over $M_{\emptyset}$ and dually, $h\left(N_{Y}\right)$ is dominated by $M_{Y}$ over $M_{\emptyset}$. Thus $h\left(N_{X}\right) \underset{M_{\emptyset}}{\downarrow} h\left(N_{Y}\right)$. But $p \in S(M)$ and $M$ is a-prime over $h\left(N_{X}\right) \cup h\left(N_{Y}\right)$. By NDOP, either $p \not \perp h\left(N_{X}\right)$ or $p \not \perp h\left(N_{Y}\right)$. As the cases are symmetric, assume $p \not \perp h\left(N_{X}\right)$, Finally, since $M$ is a-prime over $h\left(N_{X}\right) \cup M_{\omega}, h\left(N_{X}\right) \in \mathcal{N}_{X}$, so $X \in W$.

Claim 5.18. Each $W_{\Delta}$ is a $\Sigma_{1}^{1}$-subset of $\mathcal{P}(\omega)$. 
Proof. Fix $\Delta \subseteq L$ finite. Choose $C \subseteq M$ countable such that $p$ is based and stationary over $C$. Since $M$ is a-atomic over $M_{\omega}$, we can choose a countable set $Z \subseteq M_{\omega}$ such that $\operatorname{tp}(C / Z) \vdash \operatorname{tp}\left(C / M_{\omega}\right)$. Using Lemma 5.7 find a stable system $\bar{A}$ indexed by $J$ in which every $A_{u}$ is countable, $Z \subseteq A_{\omega}$, and $(\bar{A}, \bar{M})$ is a linked pair of stable systems. Note that $\operatorname{tp}\left(C / M_{\omega}\right)$ does not fork over $A_{\omega}$ by transitivity.

SubClaim 5.19. $X \in W_{\Delta}$ if and only if there exist a countable $\bar{B}$ such that $(\bar{A}, \bar{B})$ is a linked pair of stable systems and $\operatorname{tp}\left(C / B_{\omega}\right)$ does not fork over $A_{\omega}$, a countable model $N^{\prime}$ that is pseudo $\ell$-atomic over $\bar{B}_{X}$, a countable set $D \supseteq N^{\prime} C$, a tuple $d$ from $D$, a formula $\varphi(x, y z) \in \Delta$, and a type $q \in S(D)$ that does not fork over $N^{\prime}$ such that $R_{\Delta}((p \mid D) \cup\{\varphi(x, d b)\})<R_{\Delta}(p)$ for some (every) b realizing $q$.

It is easily verified that

$$
\left\{\left(N^{\prime}, \bar{B}, X\right): N^{\prime} \text { is pseudo } \ell \text {-atomic over } \bar{B}_{X}\right\}
$$

is a Borel subset of a product of Polish spaces projecting onto $\mathcal{P}(\omega)$ so the $\Sigma_{1}^{1}$-ness of $W_{\Delta}$ is an immediate consequence of the subclaim.

To establish the subclaim (and hence the claim) first suppose that $X \in$ $W_{\Delta}$. Choose $N \in \mathcal{N}_{X}$ such that $p \not \not^{\Delta} N$. Choose $D_{0}, d, \varphi$, and $q_{0} \in S\left(D_{0}\right)$ from Definition 2.15 witnessing this. Choose a countable $N^{\prime} \preceq N$ such that $q_{0}$ is based on $N^{\prime}$. Since $N^{\prime}, C$ and the language $L$ are countable, we can find a countable subset $D \subseteq D_{0}$ containing $N^{\prime} C d$ and $q \in S(D)$ parallel to $q_{0}$ such that $R_{\Delta}((p \mid D) \cup\{\varphi(x, d b)\})<R_{\Delta}(p)$ for any $b$ realizing $q$. Since $N^{\prime}$ is a-atomic over $M_{X}$, it is also pseudo $\ell$-atomic over $\bar{M}_{X}$ by Lemma 5.13. Choose $E \subseteq M_{X}$ countable so that for all finite tuples $e$ from $N^{\prime}$, all $\varphi(x, y)$ and all $u \in J \cap \mathcal{P}(X)$ there is an $L(E)$-formula $\psi(x) \in \operatorname{tp}\left(e / M_{X}\right)$ that decides $\varphi\left(x, M_{u}\right)$.

Now arguing as in Lemma 5.7 there is a stable system $\bar{B}$ indexed by $J$ such that each $B_{u}$ is countable, $E \subseteq B_{X}, A_{u} \preceq B_{u} \preceq M_{u}$, and $(\bar{A}, \bar{B})$ is a linked pair of stable systems. Since $C \underset{A_{\omega}}{\downarrow} M_{\omega}$, we have $C \underset{A_{\omega}}{\downarrow} B_{\omega}$. By our choice of $E, N^{\prime}$ is pseudo $\ell$-atomic over $\bar{B}_{X}$.

Conversely, fix $X \in \mathcal{P}(\omega)$ and assume $\bar{B}, N^{\prime}, D, d, \varphi$, and $q$ are as in the subclaim. It follows immediately from Definition 2.15 that $p \not \perp^{\Delta} N^{\prime}$. By Proposition 5.9 there is an elementary map $f: \bar{B} \rightarrow \bar{M}$ such that $f \mid A_{\omega}=$ id and $f\left(B_{u}\right) \subseteq M_{u}$ for each $u \in J$. Since $\operatorname{tp}\left(C / M_{\omega}\right)$ does not fork over $A_{\omega}$ and since $A_{\omega}$ is a model, $\operatorname{tp}\left(B_{\omega} / C A_{\omega}\right)=\operatorname{tp}\left(f\left(B_{\omega}\right) / C A_{\omega}\right)$. Let $\sigma$ be an automorphism of $\mathfrak{C}$ extending $f$ that fixes $C A_{\omega}$ pointwise. Since $p$ is based and stationary over $C$, its parallelism class is invariant under the action of $\sigma$. Thus, by replacing the given $\bar{B}$ by $f(\bar{B}), p$ by $\sigma(p)$, and $N^{\prime}$ by $\sigma\left(N^{\prime}\right)$, we may assume that $B_{u} \subseteq M_{u}$ for all $u \in J$ while preserving $p \not \perp^{\Delta} N^{\prime}$. 
Now fix an enumeration $\left\langle a_{n}^{\prime}: n \in \omega\right\rangle$ of $N^{\prime}$. Since both $N^{\prime}$ and $B_{X}$ are countable and since $M$ realizes every a-isolated type over $M_{X}$, the existence theorem for a-isolated types allows us to find $N^{\prime \prime}=\left\langle a_{n}^{\prime \prime}: n \in \omega\right\rangle$ from $M$ such that $\left\langle a_{n}^{\prime \prime}: n \in \omega\right\rangle$ is an a-construction sequence over $M_{X}$ with $\operatorname{tp}\left(N^{\prime} / B_{X}\right)=\operatorname{tp}\left(N^{\prime \prime} / B_{X}\right)$. Since both $N^{\prime}$ and $N^{\prime \prime}$ are pseudo $\ell$-atomic over $\bar{B}_{X}$, Lemma $5.15(\mathrm{i})$ implies that $\operatorname{tp}\left(N^{\prime} / B_{\omega}\right)=\operatorname{tp}\left(N^{\prime \prime} / B_{\omega}\right)$. Since $M$ is $\aleph_{1}$-homogeneous, there is $C^{\prime \prime} \subseteq M$ such that $\operatorname{tp}\left(N^{\prime} C / B_{\omega}\right)=\operatorname{tp}\left(N^{\prime \prime} C^{\prime \prime} / B_{\omega}\right)$. Thus $p^{\prime \prime} \not \not^{\Delta} N^{\prime \prime}$, where $p^{\prime \prime} \in S\left(C^{\prime \prime}\right)$ is conjugate to $p$ over $B_{\omega}$. Note that $\operatorname{tp}\left(C^{\prime \prime} / M_{\omega}\right)=\operatorname{tp}\left(C / M_{\omega}\right)$ since $\operatorname{tp}\left(C / B_{\omega}\right) \vdash \operatorname{tp}\left(C / M_{\omega}\right)$. Since $M$ is $\aleph_{1^{-}}$ homogeneous over $M_{\omega}$, there is $N_{0}=\left\langle a_{n}: n \in \omega\right\rangle$ from $M$ such that

$$
C^{\prime \prime}\left\langle a_{n}^{\prime \prime}: n \in \omega\right\rangle \equiv_{M_{\omega}} C\left\langle a_{n}: n \in \omega\right\rangle
$$

Summarizing all of this, $N_{0}$ is a countable subset of $M, p \not \perp^{\Delta} N_{0}$, and $N_{0}$ is a-constructible over $M_{X}$.

Next, let $\widehat{N}=\left\langle a_{n}: n<\beta\right\rangle$ be an a-constructible model over $M_{X}$, whose construction sequence end extends $N_{0}=\left\langle a_{n}: n \in \omega\right\rangle$. By Lemma 5.15(ii), $\widehat{N}$ is an a-construction sequence over $M_{\omega}$. Let $\widehat{M}$ be a-prime over $\widehat{N} M_{\omega}$. Note that $\widehat{M}$ is also a-prime over $N_{0} M_{\omega}$. But recall that $M$ is a-prime over $M_{\omega}$ and $N_{0}$ is a countable subset of $M$. Thus $M$ is also a-prime over $N_{0} M_{\omega}$. So, by the uniqueness of a-prime models, there is an isomorphism $h: \widehat{M} \rightarrow M$ over $N_{0} M_{\omega}$. Finally, take $N=h(\widehat{N})$. Since $N_{0} \subseteq N, p \not \not^{\Delta} N$ and $M$ is a-prime over $N M_{\omega}$. Thus, $N$ witnesses that $X \in W_{\Delta}$, which completes the proof of Claim 5.18.

Claim 5.20. $p \not \perp M_{F}$ for some finite $F \subseteq \omega$.

Proof. We first argue that $W$ is not meagre. If it were, then by Lemma 5.16(i) there would be $X \subseteq \omega$ such that $X$ and $\omega \backslash X \notin W$, which would contradict Claim 5.17.

Since $W$ is not meagre, some $W_{\Delta}$ is not meagre. Fix such a $\Delta$. Since $\boldsymbol{\Sigma}_{1}^{1}$-subsets of a Polish space have the property of Baire (see, e.g., Theorem 7 of XII.8 of [3]), it follows from Claim 5.18 that there is a nonempty open subset $U_{F, G}$ of $\mathcal{P}(\omega)$ such that $U_{F, G} \backslash W_{\Delta}$ is meagre. But $U_{F, G}$ is naturally homeomorphic to $\mathcal{P}(\omega)$, so the translation of Lemma 5.16(ii) is that there are sets $\left\{X_{i}: i \in \omega\right\} \subseteq W_{\Delta}$ such that $X_{i} \cap X_{j}=F$ for all $i<j<\omega$. For each $i \in \omega$ choose $N_{i} \in \mathcal{N}_{X_{i}}$ such that $p \not \underline{L}^{\Delta} N_{i}$. Since $\bar{M}$ is a stable system, $\left\{M_{X_{i}}: i \in \omega\right\}$ is independent over $M_{F}$. Since each $N_{i}$ is a-prime over $M_{X_{i}}$ and since $M_{F}$ is a-saturated, it follows that $\left\{N_{i}: i \in \omega\right\}$ is independent over $M_{F}$. Since $p \not \not^{\Delta} N_{i}$ for each $i$, Proposition 2.16 entails that $p \not \perp M_{F}$.

To complete the proof of the theorem, fix a finite $F \subseteq \omega$ such that $p \not \perp M_{F}$. Taking $I=\{\emptyset\} \cup\{\{i\}: i \in F\}$ and $I^{*}=\mathcal{P}(F)$ in clause (iv) of Proposition 5.5, we see that $M_{F}$ is a-prime over $\bigcup\left\{M_{i}: i \in F\right\}$. As $F$ is finite, it follows from NDOP that $p \not \perp M_{i}$ for some $i \in F$ and we finish. 


\section{References}

[1] J. Baldwin, Fundamentals of Stability Theory, Springer, 1988.

[2] A. Hernandez, $\omega_{1}$-saturated models of stable theories, Ph.D. thesis, Univ. of California, Berkeley, 1992.

[3] K. Kuratowski and A. Mostowski, Set Theory, North-Holland, 1976.

[4] M. C. Laskowski, Descriptive set theory and uncountable model theory, in: Logic Colloquium '03, Lecture Notes in Logic 24, V. Stoltenberg-Hansen and J. Väänänen (eds.), Assoc. Symbol. Logic, La Jolla, CA, 2006, 133-145.

[5] M. Makkai, A survey of basic stability theory with particular emphasis on orthogonality and regular types, Israel J. Math. 49 (1984), 181-238.

[6] A. Pillay, Geometric Stability Theory, Oxford Univ. Press, 1996.

[7] S. Shelah, Classification Theory, rev. ed., North-Holland, Amsterdam, 1990.

[8] - More on frames, preprint F569.

Department of Mathematics

University of Maryland

College Park, MD 20742, U.S.A.

E-mail: mcl@math.umd.edu
Institute of Mathematics Hebrew University Jerusalem, Israel and Department of Mathematics

Rutgers University New Brunswick, NJ 08903, U.S.A.

Received 10 May 2004;

in revised form 7 November 2005 